\title{
Polyplex-loaded hydrogels for local gene delivery to human dermal fibroblasts
}

\author{
José Antonio Durán-Mota ${ }^{1,2}$, Júlia Quintanas Yani ${ }^{1,2}$, Benjamin D. Almquist ${ }^{2}$, \\ Salvador Borrós ${ }^{1, \dagger}$, Nuria Oliva ${ }^{2, \dagger}$ \\ ${ }^{1}$ Grup d'Enginyeria de Materials (GEMAT), Institut Químic de Sarrià, Universitat Ramon \\ Llull, Via Augusta 390, 08017 Barcelona, Spain \\ ${ }^{2}$ Department of Bioengineering, Imperial College London, London SW7 2AZ, United \\ Kingdom \\ †Corresponding Authors: salvador.borros@iqs.url.edu, n.oliva-jorge@imperial.ac.uk
}

KEYWORDS. hydrogel, polyethylene glycol, poly( $\beta$-amino ester $)$, nanoparticles, gene delivery, Human Dermal Fibroblasts, skin, wound healing.

\begin{abstract}
Impaired cutaneous healing, leading to chronic wounds, affects between 2 and 6\% of the total population in most developed countries and it places a substantial burden on healthcare budgets. Current treatments involving antibiotic dressings and mechanical debridement are often not effective, causing severe pain, emotional distress and social isolation in patients for years or even decades, ultimately resulting in limb amputation. Alternatively, gene therapy (such as mRNA therapies) emerges as a viable option to promote wound healing through modulation of gene expression. However, protecting the genetic cargo from degradation and efficient transfection into primary cells remain significant challenges in the push to clinical translation. Another limiting aspect of current therapies is the lack of sustained release of drugs to match the therapeutic window. Herein, we have developed an injectable, biodegradable and biocompatible hydrogelbased wound dressing that delivers pBAE nanoparticles in a sustained manner over a range of therapeutic windows. We also demonstrate that $\mathrm{pBAE}$ nanoparticles, successfully used in previous in vivo studies, protect the mRNA load and efficiently transfect human dermal fibroblasts upon sustained release from the hydrogel wound dressing. This prototype wound dressing technology can enable the development of novel gene therapies for the treatment of chronic wounds.
\end{abstract}




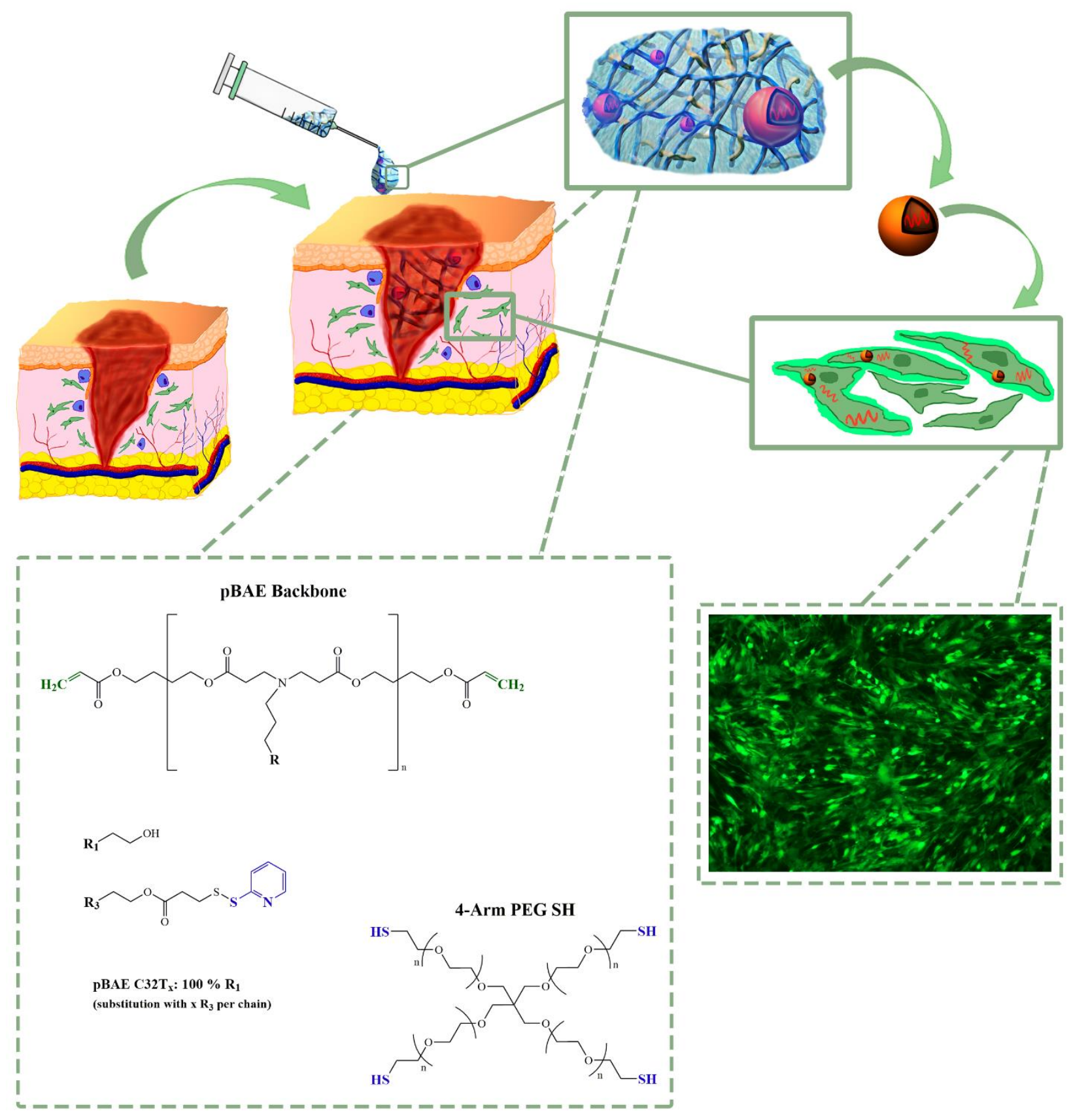

Figure 1. Schematic diagram of the composite hydrogel wound dressing designed herein for applications involving human dermal fibroblasts transfection, based on a pBAE-PEG injectable hydrogel doped with mRNA-loaded polyplexes. 


\section{INTRODUCTION}

Wound healing is a complex process involving four highly orchestrated phases ${ }^{1,2}$. Failure to complete these normal stages in a coordinated fashion leads to impaired cutaneous healing, such as delayed acute wounds and chronic wounds ${ }^{3}$. In the United States alone, over 6 million people suffer from chronic wounds, typically due to underlying conditions like obesity, diabetes or ischemia. In 2014, wound care products accounted for $\$ 2.8$ billion of the global healthcare budget, and by 2024, the advanced wound care market for surgical wounds and chronic ulcers is expected to exceed $\$ 22$ billion ${ }^{4}$. Current clinical approaches to chronic wound care are quite limited given the societal impact, and consist of approaches such as antibiotic dressings, mechanical debridement and offloading, and negative pressure therapy. When these treatments fail to work for wounds such as diabetic ulcers, many times amputation becomes necessary ${ }^{5-8}$.

Impaired wound healing has been associated with alterations in the expression of genes that mediate healing ${ }^{9-11}$, positioning mRNA delivery as an attractive therapeutic approach to restore normal protein expression and promote healing ${ }^{12}$. mRNA therapies can also be exploited to promote cells to synthesize therapeutic proteins efficiently and safely ${ }^{13,14}$. However, the delivery of nucleic acids is challenging, owing to their susceptibility to rapid degradation, clearance in biological fluids, as well as their inability to cross cytoplasmatic membranes ${ }^{15}$. Numerous vehicles have been developed over the last decade, each with their own limitations and challenges ${ }^{15,16}$. For example, viral vectors are capable of high transduction efficiency and sustained transgene expression, but they cause high levels of immunogenicity, limiting their translation to human use ${ }^{17}$. In contrast, non-viral vectors show lower transfection efficiencies than viruses, but are usually cheaper to synthesize, present better loading capacities for both DNA and RNA, and are safer for the host.

Cationic polymers, such as poly( $\beta$-amino ester)s (pBAEs), are a type of non-viral vector able to neutralize negatively charged oligonucleotides and form discrete particles, also known as polyplexes, through electrostatic interactions ${ }^{18-23}$. Polyplexes' positive overall net charge allows them to bind to cell membranes and enter the cytoplasm via endosomal transport. In addition, amines and terminal acrylates in these polymers confer the versatility of incorporating chemical groups into their structure to tune their functions and properties ${ }^{21,24}$, such as improving transfection efficiencies by conjugating endosomolytic moieties ${ }^{25}$. Moreover, pBAEs are biodegradable and biocompatible $^{26}$. In recent years, we have developed many oligopeptide-modified pBAEs polyplexes (OM-pBAEs) ${ }^{21,27}$, showing high transfection efficiency and excellent biocompatibility in different cancer cell lines ${ }^{22,28-30}$, as well as efficient in vivo transfection ${ }^{31}$, making these nanoparticles a highly promising candidate for clinical translation of new cancer therapies. However, efficient transfection of primary human cells remains a challenge, hampering the progress of new gene therapies for numerous non-cancerous pathologies, such as chronic wounds. 
Local delivery of therapeutics is often preferred over systemic delivery, as it allows for reduced dosages, enhanced stability and increased biocompatibility ${ }^{32}$. Moreover, the smart design of local delivery platforms allows for sustained and controlled release of therapeutics to injured or diseased cells, a critically important aspect in the highly dynamic process of wound healing ${ }^{33,34}$. The structure and properties of hydrogels make them optimal candidates to release therapeutic nanoparticles for wound healing ${ }^{35-37}$, maintaining a warm moist environment and allowing the absorption of wound exudates and adequate oxygen circulation, necessary to promote healing and prevent bacterial infections ${ }^{38,39}$. Moreover, hydrogels' hydrophilic nature, capable of absorbing up to $90 \%$ of water or fluids, confers them high porosity and mechanical properties resembling those of human tissues. Other characteristics such as biodegradability, biocompatibility, low immunogenicity and ease of usage have propelled their translation to the clinic ${ }^{40}$.

In the present work, we describe and characterize a new local gene delivery platform for cutaneous wound healing based on a composite synthetic hydrogel, made of pBAE and PEG polymers, doped with polynucleotide-loaded pBAE nanoparticles (Figure 1) to enable efficient transfection of human dermal fibroblasts (HDFs). Efficient transfection of HDFs is essential for developing new gene therapies for wound healing owing to their extensive involvement in the process of wound healing ${ }^{7,41}$, and their reported altered gene expression profile in chronic wounds ${ }^{42-45}$. The hydrogel developed herein is injectable, enabling in situ polymerization and high surface contact area in deep wounds with irregular topography, a typical feature of chronic wounds like diabetic foot ulcers. In the future, the versatility of pBAEs will allow for further modifications of the hydrogel network and/or the polyplexes to incorporate new and improved features to this novel wound dressing platform, such as smarter control over the release or tissue- and cell-specific transfection.

\section{MATERIALS AND METHODS}

Materials. Reagents and solvents were purchased from Sigma-Aldrich and Panreac and used as received unless otherwise stated. Oligopeptides were obtained from Ontores Biotechnologies Inc. Plasmid reporter green fluorescent protein (pmaxGFP) (3486 bp) was acquired from Amaxa, CleanCap EGFP mRNA (5moU) from Tebu-Bio, Firefly Luciferase reporter plasmid FLuc from Promega Corporation and CleanCap Fluc mRNA 5-methoxyuridine from TriLink. Human Dermal Fibroblasts (HDFs) from adult skin were purchased from ATCC (ATCC® PCS-201-030). Products for cell culture (DMEM, phosphate-buffered saline (PBS), glutamine and penicillin-streptomycin solution, trypsin-EDTA $0.25 \%$ ) were obtained from Gibco, Hyclone, and Invitrogen. ${ }^{1} \mathrm{H}-\mathrm{NMR}$ spectra were recorded in a $400 \mathrm{MHz}$ Varian (Varian NMR Instruments, Claredon Hills, IL, USA) and methanol- $\mathrm{d}_{4}$ was used as solvent unless otherwise stated. 

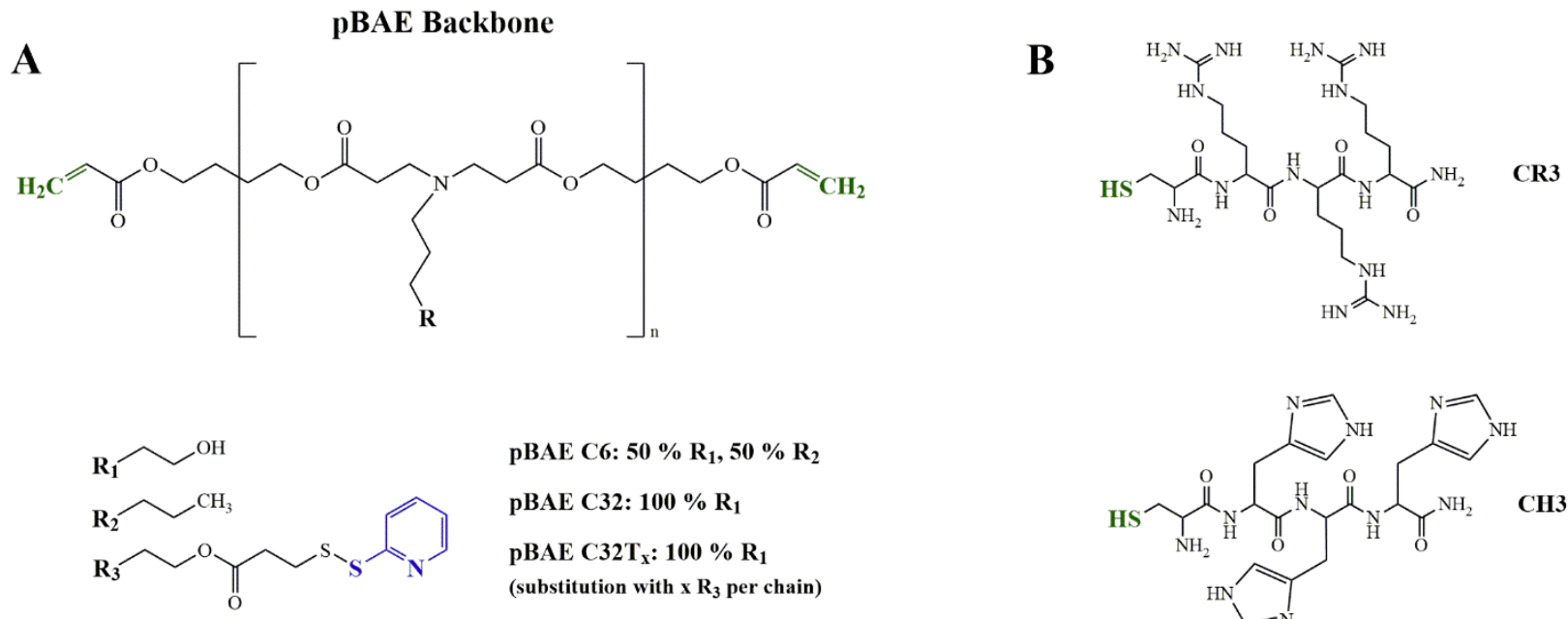

pBAE C6: $50 \% \mathrm{R}_{1}, \mathbf{5 0} \% \mathrm{R}_{2}$

pBAE C32: $100 \% \mathrm{R}_{1}$

pBAE C32T $: 100 \% \mathrm{R}_{1}$

(substitution with $\times \mathrm{R}_{3}$ per chain)

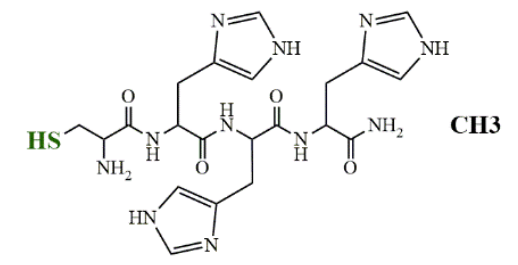

C

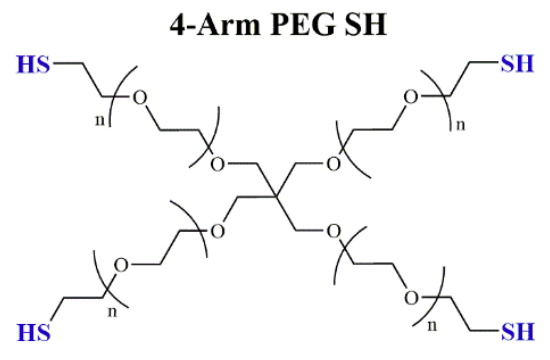

D

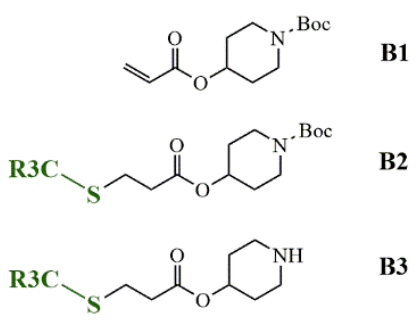

Figure 2. A) General chemical structure of pBAE polymers, where the ratio and chemical identity of R (R1 - alkyl alcohol, R2 - alkyl or R3 - thiopyridyl ester) define the nomenclature of the final product $\left(\mathrm{C} 6, \mathrm{C} 32\right.$ or $\left.\mathrm{C}_{32} \mathrm{~T}_{\mathrm{x}}\right)$. B) Chemical structure of arginine (CR3) and histidine (CH3) oligopeptides used to modify the terminal acrylates of pBAE polymers. C) Chemical structure of 4-arm PEG-SH used to crosslink $\mathrm{C}_{32} \mathrm{~T}_{\mathrm{x}}$ polymers to form the hydrogel network. D) Protecting groups used during the synthesis of the various pBAE custom polymers.

Synthesis of pBAE polymer backbones. Acrylate-terminated poly( $\beta$-aminoester)s C32 and C6 (Figure 2A) were synthesized following a procedure previously described in the literature by Dosta et al. ${ }^{27}$ Specifically, the polymer formation occurs by addition reaction of primary amines with diacrylates. C32 polymer was obtained by stirring 5-amino-1-pentanol (7.7 g, $75 \mathrm{mmol}$ ) and 1,4-butanediol diacrylate $(18 \mathrm{~g}, 82 \mathrm{mmol})$ together at $90^{\circ} \mathrm{C}$ for $20 \mathrm{~h}$. For C6 polymer, 5-amino-1pentanol (3.9 g, $38 \mathrm{mmol})$ was firstly mixed with 1-hexylamine (3.8 g, $38 \mathrm{mmol})$. Then, 1,4butanediol diacrylate $(18 \mathrm{~g}, 82 \mathrm{mmol})$ was added to the mixture and heated at $90{ }^{\circ} \mathrm{C}$ for $20 \mathrm{~h}$. Polymer backbones were characterized by ${ }^{1} \mathrm{H}-\mathrm{NMR}$ as described in our previous works ${ }^{21,31,46,47}$, and the number of repeated units of the polymer was confirmed to be $n=7$. 


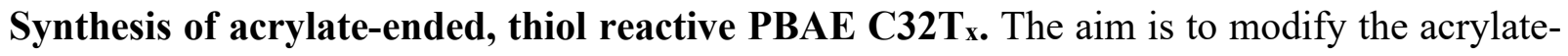
ended C32 polymer with the group 3-(2-pyridyldithio)propanoic acid (SPDP) in order to obtain a thiol-reactive C32 pBAE (Figure 2A). To prepare the SPDP acid, the first step consisted of dissolving Aldrithiol-2 (1 g, $4.46 \mathrm{mmol})$ in ethanol. The solution was purged with argon and protected from light along the process. Glacial acetic acid was added dropwise $(0.134 \mathrm{~mL})$ while stirring. Finally, 3-mercaptopropionic acid $(0.237 \mathrm{~g}, 2.23 \mathrm{mmol})$ was mixed with the previous solution. The flask was allowed to react for $2 \mathrm{~h}$ at room temperature and the final product was purified by column chromatography using basic activated $\mathrm{Al}_{2} \mathrm{O}_{3}$ as stationary phase and $\mathrm{CH}_{2} \mathrm{Cl}_{2}: \mathrm{CH}_{3} \mathrm{CH}_{2} \mathrm{OH}: \mathrm{CH}_{3} \mathrm{COOH}(60: 40: 1)$ solution as mobile phase. Then, the incorporation of SPDP groups to $\mathrm{C} 32$ chains is produced via Steglich esterification. In the present work $\mathrm{C}_{32} \mathrm{~T}_{2}$ was used. The subscript in $\mathrm{T}$ indicates the number of SPDP groups in a typical chain of seven monomer repetitions in average. Briefly, C32 PBAE $(1 \mathrm{~g}, 0.4 \mathrm{mmol})$, SPDP $(0.215 \mathrm{~g}, 1.0 \mathrm{mmol})$ and a few milligrams of 4-Dimethylaminopyridine (DMAP) were dissolved in anhydrous $\mathrm{CH}_{2} \mathrm{Cl}_{2}$. The solution was cooled at $4{ }^{\circ} \mathrm{C}$ for $30 \mathrm{~min}$ and then $\mathrm{N}, \mathrm{N}^{\prime}$-Dicyclohexylcarbodiimide (DCC) $(0.250 \mathrm{~g}$, $1.2 \mathrm{mmol}$ ) was added. The mixture was allowed to react overnight at room temperature under inert atmosphere and protected from light. Finally, the product was dissolved in acetonitrile:ethyl acetate $(1: 1)$ and kept $3 \mathrm{~h}$ at $4{ }^{\circ} \mathrm{C}$ to precipitate and separate DCC salts. $\mathrm{C}_{2} \mathrm{~T}_{2}$ polymer was characterized by ${ }^{1} \mathrm{H}-\mathrm{NMR}$. The number of SPDPs per chain was confirmed by comparing integrals of signals in terminal acrylates at $\delta=5.8-6.4 \mathrm{ppm}$ and in thiopyridyl group at $\delta=7.0-8.5 \mathrm{ppm}$ (Supplementary figure S1).

Modification of acrylate-ended pBAEs with oligopeptides. Peptides were purchased as trifluoro acetic acid salts. The first step was the substitution of trifluoro acetic acid for hydrochloride as counterions. Generally, oligopeptides (100 mg) were dissolved in $\mathrm{HCl} 0.1 \mathrm{M}$ (10 $\mathrm{mL}$ ) and frozen at $-80^{\circ} \mathrm{C}$ for an hour. The solution was then freeze-dried. Oligopeptides used in the present work were Cys-Arg-Arg-Arg (CR3) and Cys-His-His-His (CH3) (Figure 2B). Peptides hydrochlorides were reacted with acrylate-ended C32 or C6 polymers following a Michael-type addition at a pBAE:peptide molar ratio of 1:2.5. PBAEs and peptides were dissolved separately in dimethyl sulfoxide (DMSO) at $100 \mathrm{mg} / \mathrm{mL}$ concentration. Then, polymer solution was added dropwise to the peptide solution. At this point, triethylamine was added to the solution in a peptide:triethylamine molar ratio of $1: 8$. The mixture was allowed to react at room temperature for $48 \mathrm{~h}$. The modification of $\mathrm{C}_{32} \mathrm{~T}_{2}$ to obtain $\mathrm{C}_{32} \mathrm{~T}_{2} \mathrm{CR} 3$ polymer followed another synthetic process to prevent cysteine reaction with SPDP groups in the backbone. Shortly, thiols from oligopeptides were protected with a piperidine-derived group. First, 1-Boc-4-hydroxypiperidine (100 mg, 0.50 mmol) and triethylamine $(0.115 \mathrm{~mL}, 0.80 \mathrm{mmol})$ were dissolved in $\mathrm{CH}_{2} \mathrm{Cl}_{2}$ and cooled to $0{ }^{\circ} \mathrm{C}$. Acryloyl chloride $(0.044 \mathrm{~mL}, 0.52 \mathrm{mmol})$ was added to the mixture. The solution was stirred overnight at room temperature. The product obtained (B1) was washed in a separating funnel, first with water and then with a saturated solution of sodium bicarbonate. Finally, the product B1 was dried under vacuum (Figure 2D). In the second step, B1 $(90 \mathrm{mg}, 0.24 \mathrm{mmol}$ ) and the CR3 peptide (200 mg, $0.33 \mathrm{mmol}$ ) were dissolved separately in $0.5 \mathrm{~mL}$ of DMSO and then mixed. The solution was stirred overnight at room temperature. Precipitation of the product B2 (Figure 2D) occurs 
after adding the mixture dropwise to a solution of diethyl ether:acetone (4:1) and centrifuging at $4000 \mathrm{rpm}$ for $10 \mathrm{~min}$. The final step consisted of the removal of the Boc group. B2 (100 mg, 0.110 mmol) was dissolved in a solution of TFA $(0.483 \mathrm{~mL})$ and $\mathrm{CH}_{2} \mathrm{Cl}_{2}(0.887 \mathrm{~mL})$. The mixture was stirred at room temperature for $4 \mathrm{~h}$. Then, the product obtained was dried under vacuum, dissolved in a solution of $\mathrm{CH}_{2} \mathrm{Cl}_{2}: \mathrm{CH}_{3} \mathrm{OH}(5: 1)$ and passed through an Amberlyst $\mathrm{A} 21$ column. Immediately, $\mathrm{B} 3$ product was used to react with $\mathrm{C}_{32} \mathrm{~T}_{2}$ in a polymer:B3 molar ratio of 1:2.5, without addition of triethylamine. All OM-pBAEs were characterized by ${ }^{1} \mathrm{H}-\mathrm{NMR}$ as described in our previous works ${ }^{21,31,46,47}$.

PBAE polyplexes preparation. Oligopeptide-modified C6 and C32 pBAE nanoparticles were prepared following protocols based on our previous works ${ }^{21,27,47}$. Polymers used were C6CR3, C6RH (C6CR3:C6CH3 in a 6:4 ratio) and C32CR3. Polynucleotides used in transfections were plasmid reporter green fluorescent protein (pGFP), EGFP mRNA (mRNA-GFP), firefly luciferase reporter plasmid (pFLuc) and mRNA-FLuc. PBAEs and polynucleotides were kept in stocks at $100 \mathrm{mg} / \mathrm{mL}$ in DMSO or $1 \mathrm{mg} / \mathrm{mL}$ in nuclease-free water, respectively. For polyplexes formation, these starting solutions were diluted separately in sodium acetate ( $\mathrm{AcONa}$ ) $\mathrm{pH}=5.2$ buffer. The concentration of AcONa salts used was $12.5 \mathrm{mM}$ for $\mathrm{C} 6$ or $25 \mathrm{mM}$ for $\mathrm{C} 32$. The final volume of the $\mathrm{pBAE}$ and the polynucleotide solutions was the same and it was calculated to reach a polynucleotide final concentration of $0.03 \mathrm{mg} / \mathrm{mL}$ and the.desired pBAEs:polynucleotide weight ratio when mixed. This ratio was 25:1 for $\mathrm{C} 6$ and 50:1 for $\mathrm{C} 32$. In relation to the mixing process, the polynucleotide solution was added over the PBAE solution by pipetting and incubated at 25 ${ }^{\circ} \mathrm{C}$ for $30 \mathrm{~min}$. Analysis of particle size distribution was performed in a Nanosizer ZS instrument (Malvern Instruments, UK) diluting polyplexes in a 10-fold volume of phosphate-buffered saline (PBS 1x).

Preparation of PEG-pBAE hydrogels. Hydrogel matrix formation occurs by the crosslinking of 4arm-PEG-SH molecules $\left(\mathrm{M}_{\mathrm{n}}=5000\right)$ with $\mathrm{C} 32 \mathrm{~T}_{2} \mathrm{CR} 3$ pBAE (Figure 2C) in different PEG:pBAE ratios. The presence of SPDP in the $\mathrm{C}_{32} \mathrm{~T}_{2} \mathrm{CR} 3$ polymer allows the chemical crosslinking with thiols in the PEG polymer, hence forming the hydrogel in situ. For its preparation, the 4arm-PEG-SH and $\mathrm{C}_{3} 2 \mathrm{~T}_{2} \mathrm{CR} 3$ were separately dissolved in DMSO at a concentration of $500 \mathrm{mg} / \mathrm{mL}$ and $250 \mathrm{mg} / \mathrm{mL}$ respectively. The pBAE solution was added over the PEG solution to achieve the desired PEG:pBAE ratio after mixing. The solution was mildly shaken and incubated at room temperature for 30 minutes. Finally, each sample was washed with deionized water five times in order to fully eliminate DMSO traces. Hydrogels used in the present work had a PEG:pBAE molecules ratios of 1:1 (HG11, one PEG is crosslinked with one linear $\mathrm{C}_{32} \mathrm{~T}_{2} \mathrm{CR} 3$ ) and 1:4 (HG14, a PEG molecule is crosslinked using four $\mathrm{C}_{2} 2 \mathrm{~T}_{2} \mathrm{CR} 3 \mathrm{pBAE}$ ).

Preparation of PEG-pBAE hydrogels doped with pBAE nanoparticles. Following the $p B A E$ polyplexes preparation section detailed above, $4 \mu \mathrm{L}$ of $\mathrm{pBAE}$ nanoparticles solution at $0.3 \mathrm{mg} / \mathrm{mL}$ polynucleotide concentration were prepared. Before forming the hydrogels, nanoparticles solutions were first mixed with the PEG solution. Then, PBAEs solution was added to the mixture 
and the preparation of PEG-pBAE hydrogels protocol described above was followed without changes. To further understand the hydrogels behaviour, the two formulations studied in the present work were prepared with or without nanoparticles and using different concentrations and final volumes (Table 1).

\begin{tabular}{|c|c|c|c|c|c|c|c|}
\hline Formulation & {$[P E G]_{i}$} & $V_{P E G}$ & {$[p B A E]_{i}$} & $\mathbf{V}_{\mathbf{p B A E}}$ & $\mathbf{V}_{\mathrm{NP}}$ & V DMSO & $\mathbf{V}_{\mathbf{f}}$ \\
\hline$H G 11$ & 500 & 8 & 250 & 8 & 0 & 0 & 16 \\
\hline$H G 14$ & 500 & 8 & 250 & 32 & 0 & 0 & 40 \\
\hline HG11-NP & 500 & 8 & 250 & 8 & 4 & 0 & 20 \\
\hline$H G 14-N P$ & 500 & 8 & 250 & 32 & 4 & 0 & 44 \\
\hline$H G 14-N P_{1 / 2}$ & 500 & 8 & 250 & 32 & 2 & 2 & 44 \\
\hline$H G 11-500 v$ & 500 & 8 & 500 & 4 & 0 & 12 & 24 \\
\hline$H G 14-500 v$ & 500 & 8 & 500 & 16 & 0 & 0 & 24 \\
\hline$H G 11-N P-500 v$ & 500 & 8 & 500 & 4 & 4 & 12 & 28 \\
\hline$H G 14-N P-500 v$ & 500 & 8 & 500 & 16 & 4 & 0 & 28 \\
\hline
\end{tabular}

Table 1: Summary of initial concentrations, volumes and final volumes of the different hydrogel formulations studied in the present work. Concentration is given in $\mathrm{mg} / \mathrm{mL}$ and volumes in $\mu \mathrm{L}$.

Confocal microscopy characterization of PEG-pBAE hydrogels. Microstructure of hydrogels and polyplex-loaded hydrogels were studied by confocal fluorescence microscopy. For pore size and distribution studies, the hydrogels were prepared using $0.5 \%$ of the $\mathrm{C}_{3} \mathrm{~T}_{2} \mathrm{CR} 3$ polymer forming the hydrogel matrix tagged with fluorescein isothiocyanate (FITC). Hydrogels were immersed in optimal cutting temperature (OCT) compound, frozen at $-80{ }^{\circ} \mathrm{C}$ overnight and 25 and $50 \mu \mathrm{m}$ thickness slices obtained with a cryotome. For studies of polyplexes distribution, hydrogels loaded with pBAE nanoparticles were prepared following the protocol described in the previous section. Specifically, pGFP-loaded C6RH polyplexes were embedded inside the hydrogels, where $2 \%$ of C6RH polymer was labelled with cy5 dye and $10 \%$ of pGFP was labelled with cy3 dye to image both the nanoparticle and the polynucleotide cargo and study dye colocalization as a surrogate for nanoparticle stability in the hydrogel structure. Hydrogels were immersed in distilled water for 30 min to wash nanoparticles that may be weakly adsorbed on the hydrogel surface prior to imaging. Images were taken using a Leica SP8 confocal microscope (Leica Microsystems). Depending on the experiment, FITC, cy3 and cy5 wavelengths were 
selected using the microscope software. Image processing, pore size distribution, analysis and colocalization studies were done with ImageJ-Fiji software.

Sample preparation for scanning electron microscopy (SEM): Hydrogels formulations with and without nanoparticles were prepared following the protocol described in the previous section. Samples were frozen at $-80^{\circ} \mathrm{C}$ overnight and freeze-dried prior to imaging with a SEM. No sputter coating was used for the visualization of the samples.

Hydrogels swelling. Duplicate of samples HG11 and HG14 were prepared following the protocol given above. Different drying processes were followed in parallel to compare hydrogel behaviour. In the first method, samples were dried for $24 \mathrm{~h}$ in a lab oven at $37^{\circ} \mathrm{C}$. Alternatively, samples were frozen at $-80{ }^{\circ} \mathrm{C}$ overnight and a lyophilized afterwards. The residue obtained after each drying method was weighted. Hydrogels were then incubated in $1 \mathrm{~mL}$ of Milli-Q water for $30 \mathrm{~min}$. Throughout the process, the samples were slightly squeezed with tweezers to facilitate the complete entry of water into the networks. Swollen hydrogels were weighted and then the swelling ratio was calculated with the following equation:

Swelling $(\%)=\frac{\mathrm{Ws}-\mathrm{Wd}}{\mathrm{Wd}} \cdot 100(1)$

Where Ws and Wd refer to the weight of the swollen hydrogel and the dried hydrogel respectively.

Hydrogels rheological characterization. Triplicates of samples of HG11 and HG14 were freshly prepared and immediately used in the measurements. Storage $\left(G^{\prime}\right)$ and loss $\left(G^{\prime \prime}\right)$ moduli were measured as a function of the strain at $25^{\circ} \mathrm{C}$ with Ar2000ex rheometer (TA Instruments) using $8 \mathrm{~mm}$ Cross-Hatched plate. A different gap was set depending on the sample, but always setting a normal force of $0.1 \mathrm{~N}$.

Hydrogels degradation times. Hydrogels HG11 and HG14 were prepared following the protocol described previously, and $\mathrm{C}_{32} \mathrm{~T}_{2} \mathrm{CR} 3$ was tagged with fluorescein at a concentration of $2.5 \%(\mathrm{w} / \mathrm{w})$ for HG11 and 5\% (w/w) for HG14. Duplicates of these candidates were incubated at $37{ }^{\circ} \mathrm{C}$ in $200 \mu \mathrm{L}$ PBS $(1 \mathrm{x})$. The supernatant $(200 \mu \mathrm{L})$ was completely removed to measure fluorescence intensity at each timepoint and replaced with the same volume of fresh PBS solution. The progression of the degradation was followed by tracking fluorescence loss with plate and cuvette reader Tecan Infinite 200 PRO. The percentage of hydrogel integrity was calculated based on the fluorescence intensity in each timepoint relative to the total fluorescence.

PBAE nanoparticles release. Duplicate samples of HG11 and HG14 doped with C6RH pBAE nanoparticles were prepared. These polyplexes contained pGFP labelled with cy5. Samples were placed in PBS (1x) and at each time point the supernatant $(200 \mu \mathrm{L})$ was collected to measure fluorescence intensity and the same volume was replaced with fresh PBS solution. Nanoparticle release from the hydrogel was followed by tracking fluorescence loss. The percentage of released 
nanoparticles was calculated based on the fluorescence intensity in each timepoint relative to the total fluorescence.

HDFs cells culture. HDF cell line was cultured with DMEM (4.5 g glucose/mL, without glutamine, $\mathrm{pH}=7.2$ ) supplemented with glutamine $(2 \mathrm{mM}), 1 \%$ penicillin-streptomycin mixture and $10 \%$ fetal bovine serum (FBS). Cells were grown on incubators at $37{ }^{\circ} \mathrm{C}$ with $5 \% \mathrm{CO}_{2}$ atmosphere and seeded $72 \mathrm{~h}$ before starting an experiment. HDFs cells used in every experiment were at passage number 2 .

Cytotoxicity of hydrogels' degradation products. Triplicate samples of HG11 and HG14 hydrogels were degraded in a millilitre of supplemented culture medium without FBS and incubated at $37^{\circ} \mathrm{C}$. Three aliquots of $200 \mu \mathrm{L}$ of each sample were collected at $24 \mathrm{~h}, 72 \mathrm{~h}$ and 168 $\mathrm{h}$ and replaced with fresh medium. The starting reagents used to form the hydrogels were also dissolved separately in culture medium in a quantity corresponding that used for the hydrogel preparation. Before use, $10 \%$ FBS was added to each sample. HDF cells were seeded at a density of 10,000 cells per well in a 96-well plate and were grown in contact with the collected $200 \mu \mathrm{L}$ medium containing the hydrogel degradation products or the starting reagents. Cell viability assays were performed at $24 \mathrm{~h}$ using Presto Blue reagent (Invitrogen) following the manufacturer's instructions. Briefly, presto blue reagent was added and incubated for $30 \mathrm{~min}$ at $37{ }^{\circ} \mathrm{C}$. Fluorescence intensity was measured then at $540 \mathrm{~nm}$ excitation and $590 \mathrm{~nm}$ emission wavelengths.

HDFs transfection with pBAE nanoparticles. HDF cells were seeded at 10,000 cells per well in 96-well plates and incubated $24 \mathrm{~h}$ at $37^{\circ} \mathrm{C}$ in $5 \% \mathrm{CO}_{2}$ atmosphere. Cells reached a confluence of $80-90 \%$ prior to performing the transfection experiments. Different compositions of pBAE nanoparticles loaded with pGFP, pFLuc, mRNA-GFP and mRNA-FLuc polynucleotides were studied in the transfection experiments. Solutions of these polyplexes were prepared in a concentration of $0.03 \mu \mathrm{g} / \mu \mathrm{L}$ as described above and 10-fold diluted in non-supplemented DMEM. Cells were transfected with $100 \mu \mathrm{L}$ of the previous solution to a final $0.3 \mu \mathrm{g} / \mathrm{well}$ dose of the polynucleotide. HDFs were incubated for $3 \mathrm{~h}$. Subsequently, transfection media was removed, and fresh supplemented media was added to the cells. Polyplus-transfection JetPrime and JetMESSENGER were used as positive control in DNA and RNA experiments, respectively. The concentration used was that recommended by the manufacturer, which corresponds to a lower concentration than that used for $\mathrm{pBAE}$ nanoparticles due to the toxicity of JetPrime and JetMESSENGER. Untreated cells were used as negative controls. After $24 \mathrm{~h}$ incubation in the case of mRNA and $48 \mathrm{~h}$ for plasmid DNA, cells were imaged with a fluorescence microscope (Nikon Eclipse T32000-U). For quantitative measurements, cells were detached by incubating for $5 \mathrm{~min}$ with trypsin-ethylenediaminetetraacetic acid at $37{ }^{\circ} \mathrm{C}$ in $5 \% \mathrm{CO}_{2}$ atmosphere. Transfection efficiency was measured by flow cytometry (FACS; NovoCyte Flow Cytometer, ACEA Biosciences Inc.). In case of using FLuc reporters, luciferase activity was quantified using the Luciferase Assay System Kit (Promega), and photon emission was measured in a Synergy HT luminometer (BioTek). Cell viability of the formulations studied was performed using Presto Blue 
reagent and following the manufacturer's instructions. Negative control consisted of untreated cells and positive control consisted of treating cells with a highly cytotoxic solution.

HDFs transfection with hydrogels doped with pBAE nanoparticles. HDF cells were seeded at 40,000 cells per well in 48 -well plates and incubated $24 \mathrm{~h}$ at $37{ }^{\circ} \mathrm{C}$ in $5 \% \mathrm{CO}_{2}$ atmosphere. Triplicate samples of HG41 and HG44 containing mRNA-GFP-loaded C6RH pBAE polyplexes were prepared as described above. Hydrogels were washed with supplemented DMEM five times to eliminate DMSO traces and placed on top of cells together with $250 \mu \mathrm{L}$ of supplemented medium. Negative control consisted of the same hydrogels incorporating C6RH loaded with noncoding DNA segments. After $24 \mathrm{~h}$ incubation, cells were imaged with a fluorescence microscope. Transfection efficiency was measured afterwards by flow cytometry in the same conditions as mentioned in the previous section. Cell viability assay was performed after $24 \mathrm{~h}$ treatment with the formulations studied using Presto Blue reagent and following the manufacturer's instructions. Negative control consisted of untreated cells and positive control consisted of treating cells with a highly cytotoxic solution.

Statistical Analysis. GraphPad Prism 8.0.1 software was used for the statistical analysis. Statistical differences between groups were studied by ordinary one-way ANOVA with post-hoc Tukey HSD test. The significance of the difference in the data is $* p<0.05, * * p<0.01, * * * p<$ 0.001 , and $* * * * \mathrm{p}<0.0001$.

\section{RESULTS AND DISCUSSION}

The platform developed here consists of a biocompatible, biodegradable and injectable PEG:pBAE hydrogel for the local and sustained delivery of mRNA-loaded pBAE nanoparticles to transfect HDFs. We exploited the chemical versatility of pBAEs to optimize the polyplex formulation to transfect primary dermal fibroblasts. We further exploited the versatility of pBAEs by using these polymers as the hydrogel backbone that protects the nanoparticles and allows their sustained release over time.

Synthesis and characterization of oligopeptide end-modified pBAEs. pBAEs present high tunability and versatility. The polymer backbone's chemical structure plays a decisive role in the formation of polyplexes and their behaviour as transfection agents. In this work, we synthesized two families of pBAE polymers with varying polarity by controlling the molar stoichiometry of amine groups, with the $\mathrm{C} 32$ polymer being more hydrophilic than the C6 polymer (Figure 2A). To enable crosslinking of the pBAE polymers with 4arm-PEG-SH molecules, we synthesized thiol-reactive $\mathrm{C} 32$ pBAEs $\left(\mathrm{C}_{2} 2 \mathrm{~T}_{\mathrm{x}}\right)$, with $\mathrm{x}$ being approximately two thiopyridyl groups per chain (Figure 2A, Supplemental figure S1). Finally, the different pBAE acrylate-ended backbones (C6 $\mathrm{C} 32$ and $\mathrm{C}_{32} \mathrm{~T}_{2}$ ) were modified with cysteine-terminated $\mathrm{CR} 3$ or $\mathrm{CH} 3$ oligopeptides through Michael-type addition (Figure 2B, Supplemental figure S2). All ${ }^{1} \mathrm{H}-\mathrm{NMR}$ spectra of polymers were in agreement with previously published data ${ }^{27,47}$. 
A

\begin{tabular}{ccc}
\hline $\begin{array}{c}\text { Nanoparticle } \\
\text { formulation }\end{array}$ & $\begin{array}{c}\text { Hydrodynamic diameter } \\
\text { (nm; by DLS) }\end{array}$ & $\begin{array}{c}\text { PdI } \\
\text { (by DLS) }\end{array}$ \\
\hline$C 32 C R 3$ & $187.40 \pm 13.74$ & $0.123 \pm 0.032$ \\
\hline$C 6 C R 3$ & $131.63 \pm 3.72$ & $0.201 \pm 0.016$ \\
\hline$C 6 R H$ & $126.97 \pm 0.93$ & $0.154 \pm 0.006$
\end{tabular}

B

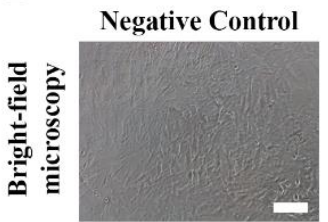

Positive Control

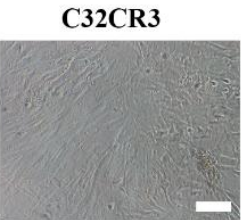

C6CR3
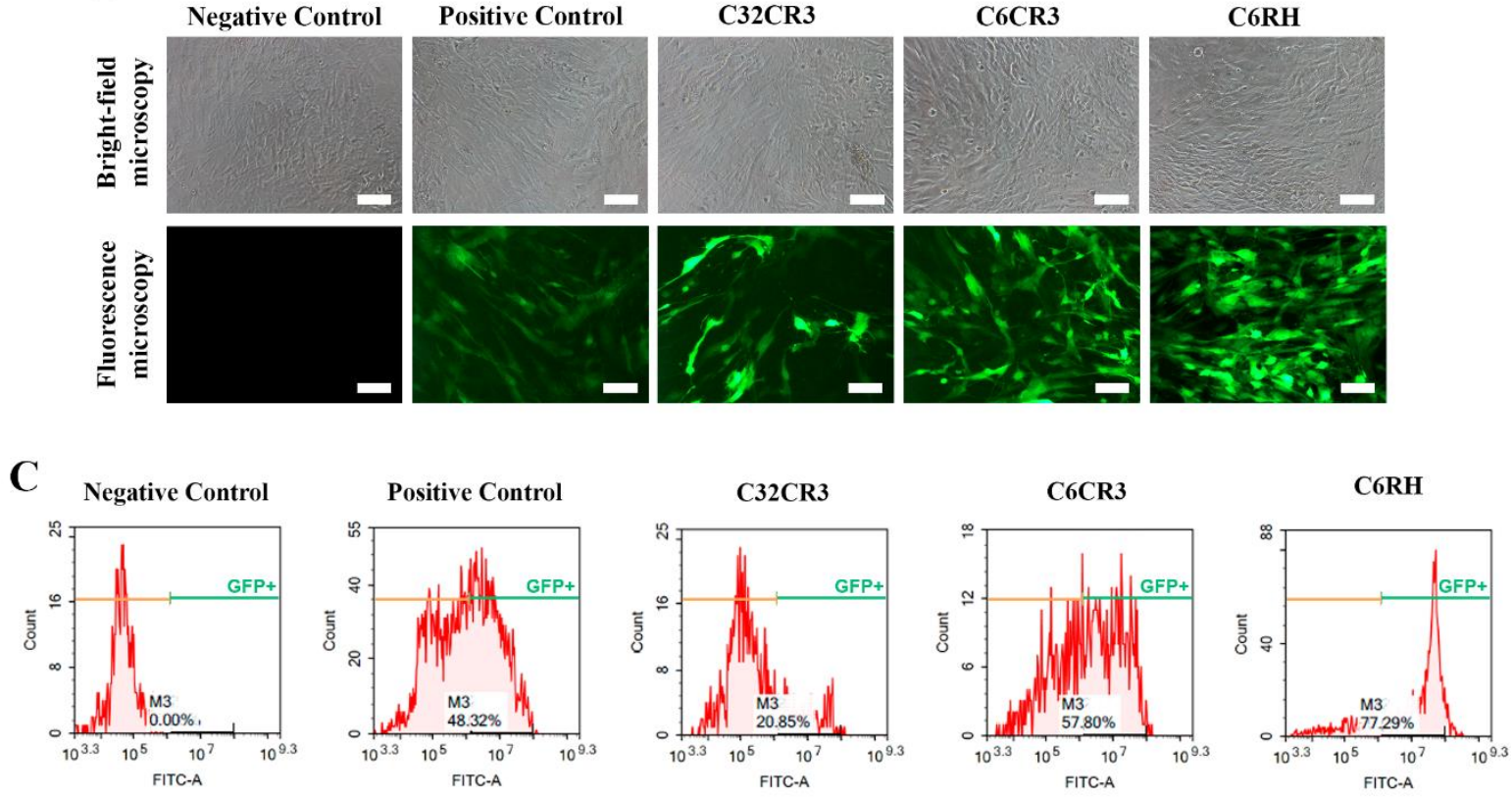

D

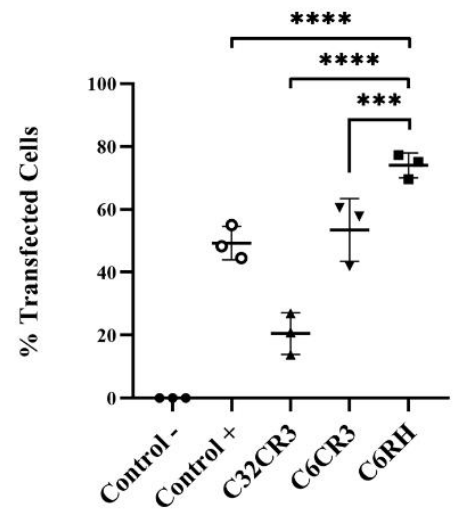

$\mathbf{E}$

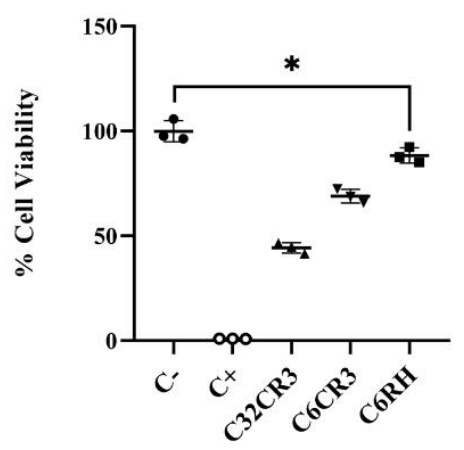

Figure 3. A) Hydrodynamic diameter and PdI of the different pBAE formulations (C32CR3, C6CR3 or C6RH) containing GFP-coding mRNA obtained by DLS technique. B) Bright-field and fluorescence microscopy images of HDF cell line expressing GFP after transfection with commercially available jetMESSENGER or different pBAE formulations containing mRNA. C) FACS graphs showing the percentage of the events counted emitting radiation at FITC wavelength. D) Quantification of transfected cells (in \%) by FACS with the different formulations 
encapsulating GFP-coding mRNA. Imaging and quantification assays were performed $24 \mathrm{~h}$ after transfection. E) Cell viability (in \%) after $24 \mathrm{~h}$ transfection using the mRNA-GFP-loaded polyplexes formulations studied. Scale bar: $100 \mu \mathrm{m} . \mathrm{n}=3$. ${ }^{*} \mathrm{p}<0.05 ;{ }^{*} \mathrm{p}<0.01 ; * * * \mathrm{p}<0.001$; $* * * * \mathrm{p}<0.0001$.

Optimization, characterization and transfection efficiency of OM-pBAE polyplexes. Several cancer cell lines have been successfully transfected using a wide variety of pBAE formulations ${ }^{21,27,46}$. However, transfection of primary human cells, such as fibroblasts, is challenging and typically yields very low transfection efficiency. We have based our current study on previously observed patterns that yielded optimal transfection efficiency in cancer cell lines, and systematically altered polyplexes formulation to tune the hydrophobicity/hydrophilicity ratio and the ability to escape the endosome, to maximize fibroblast transfection. Past research has shown that hexyl groups in polyplexes enhance endocytosis and transfection efficiency ${ }^{48,49}$ but decrease nanoparticle stability ${ }^{27}$. Alternatively, the use of alcohol pendant groups combined with hydrocarbon chains overcomes the stability limitations and affords efficient transfection (C6 polymers, Figure 2A).

Regarding endosomal escape, our previous research showed that the addition of histidine residues presents the best buffering capacity despite low encapsulation of genetic material ${ }^{21}$. On the other hand, arginine-ended OM-pBAEs showed higher encapsulation efficiency with lower endosomal escape capacity. Polyplexes formed by a mixture of equal ratios of both polymers C32CR3 and $\mathrm{C} 32 \mathrm{CH} 3$ (1:1) led to synergistic transfection efficiencies. Based on these studies, here we hypothesized that a mixture of arginine-ended (CR3) and histidine-ended (CH3) C6 pBAE polymers, which are more hydrophobic than previously studied C32 polymers (Figure 2B), would maximize oligonucleotide encapsulation, enable cellular membrane crossing and facilitate endosomal escape in primary HDFs ${ }^{27}$. Hybrid C6CR3:C6CH3 polyplexes with a 3:2 molar ratio (named C6RH from now on) showed efficient encapsulation of GFP-mRNA into nanoparticles of similar size and polydispersity index to its predecessors (C32CR3 and C6CR3, Figure 3A), previously proven to be optimal for cellular uptake $\mathrm{s}^{50,51}$ and in vivo use ${ }^{52-55}$. C6RH can also encapsulate other genetic material, such as plasmid DNA, and genetic material encoding other proteins, such as luciferase (Supplemental Table 1). C6CH3- and C32CH3-only polyplexes were not investigated owing to near-null transfection rates observed in the past $^{21}$.

Transfection efficiency was evaluated $24 \mathrm{~h}$ post-transfection with GFP-mRNA polyplexes compared to controls. GFP expression in cells was observed by fluorescence microscopy (Figure 3B) before its quantification by flow cytometry (FACS, Figure 3C-D). Overall, C6RH polyplexes demonstrated superior GFP-mRNA transfection efficiency in HDFs than previously developed formulations (C32CR3 and C6CR3) and a commercially available transfection reagent (Figure 3B-D). These results suggest that by tuning the pBAE backbone's hydrophobicity and the oligopeptide modification ratios, $\mathrm{C} 6 \mathrm{RH}$ polyplexes can cross HDFs membranes more readily and successfully escape the endosome, leading to an overall enhancement of transfection efficiency 
and reporter protein expression, while eliciting minimal toxicity (Figure 3E). Interestingly, the levels of plasmid DNA expression after transfection with C6RH polyplexes were also higher than those of previous formulations and a commercially available control (Supplementary Figure S3), indicating that $\mathrm{C} 6 \mathrm{RH}$ nanoparticles have an enhanced ability to cross the nuclear membrane and deliver genetic material to the nucleus, a unique feature for these polyplexes. To confirm this approach's broad applicability, we verified that these polyplexes successfully deliver mRNA and plasmid DNA encoding other proteins (such as luciferase) (Supplementary Figure S4). All in all, C6RH polyplexes emerge as new candidates for future applications as gene delivery vehicles due to their versatility, high transfection efficiency and low toxicity, and are suitable for the delivery of both DNA and RNA to primary human dermal fibroblasts. 
A

B

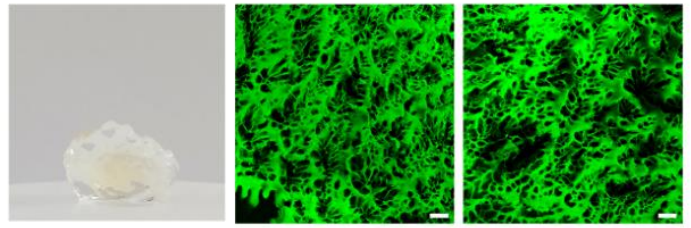

$\mathbf{E}$

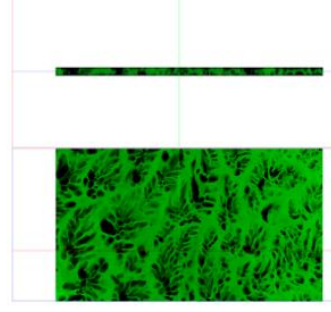

G
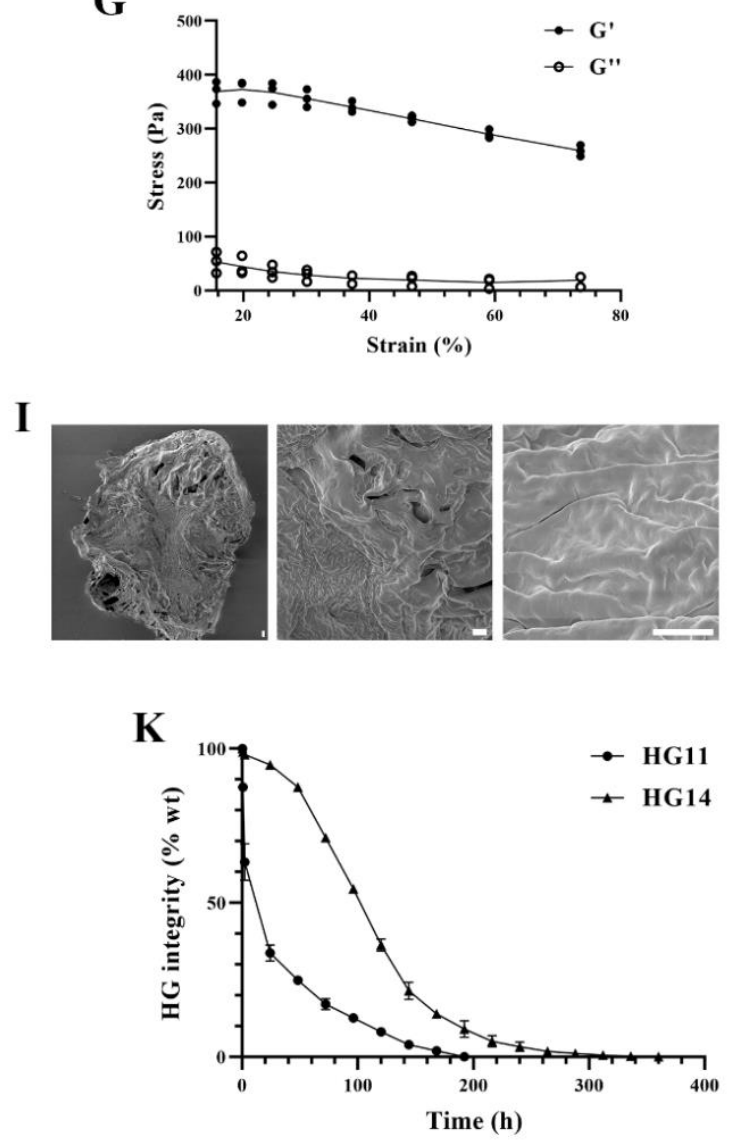

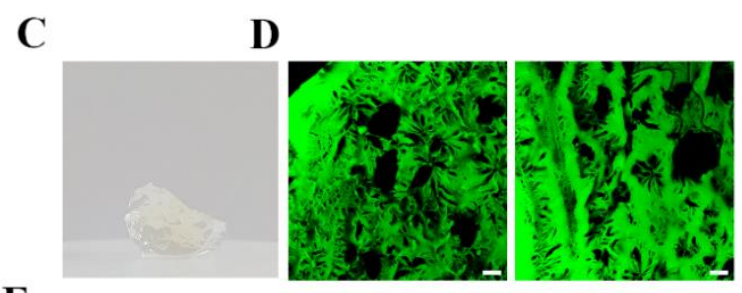

$\mathbf{F}$

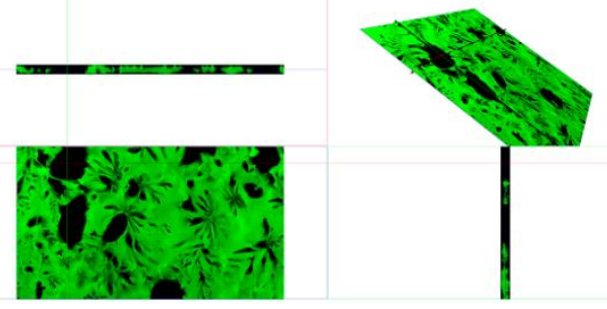

H
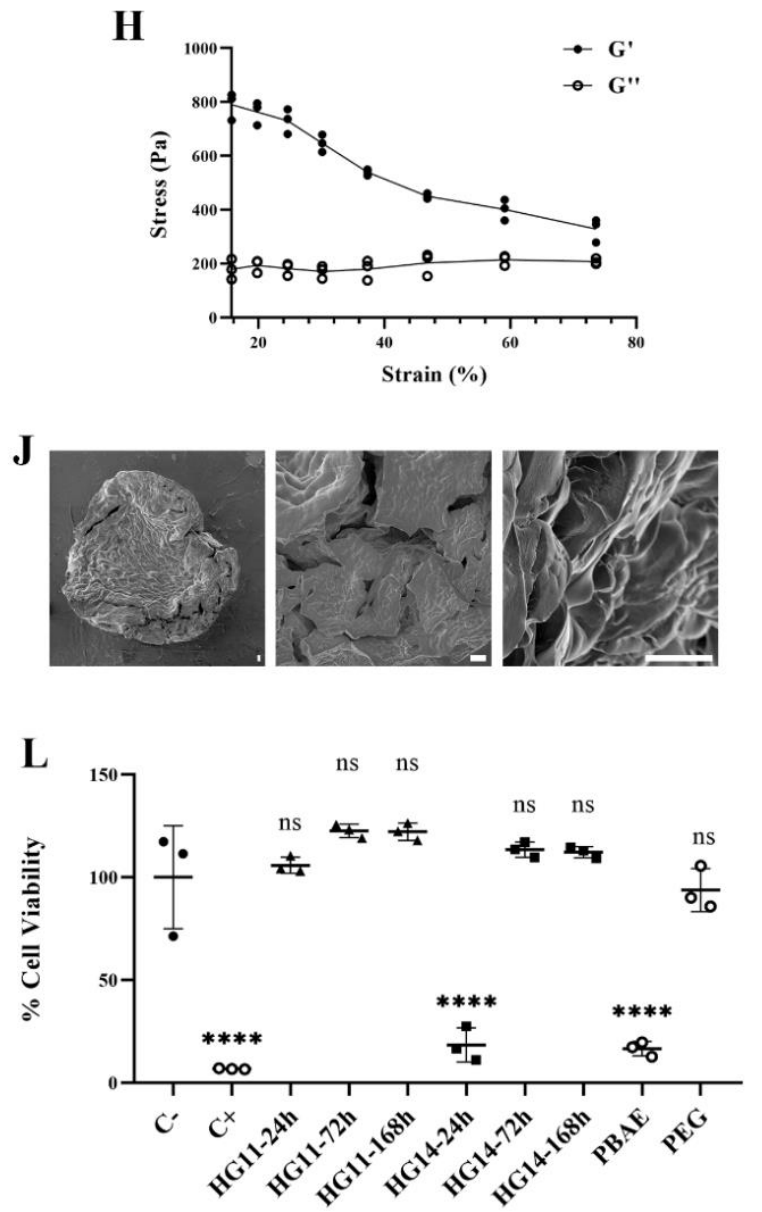

Figure 4. A) Image of hydrogel formulation HG11. B) Confocal microscopy images of $50 \mu \mathrm{m}$ thickness slices from the HG11 hydrogel tagged with FITC. C) Image of hydrogel formulation HG14. D) Confocal microscopy images of $50 \mu \mathrm{m}$ thickness slices from the HG14 hydrogel tagged with FITC. E) and F) 3D reconstruction of formulations HG11 and HG14, respectively. G) and H) Stress-strain curve showing the evolution of G' and G" for HG11 and HG44 hydrogel formulations, respectively; $\mathrm{n}=2$. I) and J) SEM images (SEM HV: $1 \mathrm{kV}$ ) of bulk lyophilized HG11 and HG14, 
respectively. K) Degradation of hydrogels HG11 and HG14 was tracked using fluorescently labelled pBAE, which was converted to weight $\%$ of pBAE in the hydrogel as a measure of hydrogel integrity; $n=2$. L) Viability of HDFs after 24 hours in contact with medium containing the degradation by-products released from the hydrogels during three time intervals $(0-24 \mathrm{~h}, 24-$ $72 \mathrm{~h}$ and $72-168 \mathrm{~h}$ ). Confocal microscopy images scale bar: $100 \mu \mathrm{m}$; SEM scale bar: $50 \mu \mathrm{m} . \mathrm{n}=$ 3. ${ }^{*} \mathrm{p}<0.05 ;{ }^{* *} \mathrm{p}<0.01 ;{ }^{* * *} \mathrm{p}<0.001 ; * * * * \mathrm{p}<0.0001$.

Preparation and characterization of PEG:PBAE hydrogels. Following the successful development of $\mathrm{C} 6 \mathrm{RH}$ polyplexes, we next explored incorporating them into a degradable hydrogel to facilitate localized, controlled delivery. We chose C32CR3 pBAEs to form the hydrogel's backbone due to their hydrophilicity ( $100 \%$ alcohol pendant groups), as well as their biodegradability and biocompatibility. C32CR3 pBAEs were chemically modified to make them thiol-reactive $\left(\mathrm{C}_{32} \mathrm{~T}_{2} \mathrm{CR} 3\right)$ and star-shaped 4arm-PEG-SH was used as a crosslinker to form the hydrogel network in situ (Figure 1 and Figure 4A, C). The formation of the hydrogel network was monitored by the disappearance of the leaving group pyridine-2-thione signals in the ${ }^{1} \mathrm{H}-\mathrm{NMR}$ spectrum (Supplementary Figure S5).

By controlling the PEG:pBAE ratio and crosslinking density, mechanical properties of the hydrogel can be readily tuned. We explored two PEG:pBAE molar ratios in the present work: 1:1 (HG11), where thiol groups are in 2-fold excess of thiol-reactive groups and 1:4 (HG14), with a 2 -fold excess of thiol-reactive groups compared to thiol groups. We studied the impact of these different ratios on the hydrogel's properties to establish our material's optimal formulation. Interestingly, the swelling ratio was not affected by the differences in PEG-pBAE ratio (Supplemental Figure S6), suggesting that both samples have similar crosslinking density and pore size. Fluorescence microscopy (Figures 4B, D, E and F) showed average pore sizes of 17.9 $\mu \mathrm{m}$ and $17.6 \mu \mathrm{m}$ for HG11 and HG14, respectively (Supplemental Figure S7). These data correlate with the similar swelling ratios and confirms that the crosslinking density (overall number of chemical bonds) is statistically similar for both formulations, as the ratio between chemical groups is maintained constant (SH:SSPy ratios of 2:1 for HG11 and 1:2 for HG14). It is important to highlight, though, that while the average crosslinking density may be statistically similar, the pore size distribution shows striking differences, with HG11 presenting a lower distribution of pore sizes ranging between 5 and $100 \mu \mathrm{m}$, while the pore size of HG14 ranges from 1 to almost $1000 \mu \mathrm{m}$ (Supplemental Figure S7). While there is no difference in swelling ratio between both formulations for a given drying protocol (oven or lyophilization), the drying method itself has a considerable influence on the swelling ratio, with approximately $400 \%$ increase when the hydrogel is lyophilized compared to oven-dried (Supplemental Figure S6). Lyophilisation can extract water more efficiently than drying at $37{ }^{\circ} \mathrm{C}$, as well as the DMSO used as the stock solutions solvent, hence leading to larger increases in re-swelling ratios.

Rheological studies revealed that the storage moduli $\left(\mathrm{G}^{\prime}\right)$ for both formulations were higher than the loss moduli (G"), indicating that these hydrogels store energy elastically and hence behave as 
viscoelastic gels. Interestingly, despite both formulations having similar swelling ratios and crosslinking density, they showed stark differences in their behaviour under deformation. HG11 formulation shows a linear viscoelastic region at low strain percentages not observed in HG14 formulation, with $G^{\prime}$ being independent of the applied deformation. As the percentage of strain increases, G' of HG11 slowly decreases as the network gradually deforms and the polymeric chains rearrange to minimize the effect of the stress applied, while G" slightly increases (Figure 4G). In comparison, $\mathrm{G}^{\prime}$ values of $\mathrm{HG} 14$ decrease more abruptly and $\mathrm{G}^{\prime \prime}$ show a relative minimum at $30 \%$ strain (Figure 4H), suggesting the development of microfractures. This behaviour is likely due to the large pores observed in fluorescence microscopy, facilitating points of increased stress levels that enabled fractures to be created and propagated. As a result, the energy that can be stored elastically $\left(G^{\prime}\right)$ falls drastically during these events, making these hydrogels more brittle and likely to break during mechanical deformation. This conclusion was further confirmed when studying the lyophilized hydrogels by SEM, where HG11 can resist the lyophilization process, in contrast to HG14 that exhibits several internal fractures (Figures 4I-J). Hence, the HG14 formulation is less elastic and more brittle than HG11.

Degradation and cytotoxicity of PEG:pBAE hydrogels. Hydrogel degradation was tracked using fluorescence, with the integrity of the hydrogel plotted as the ratio of the remaining fluorescence to the total initial fluorescence (Figure 4K). HG14 completely degraded after roughly 15 days, compared to 8 days for HG11. The burst release was more pronounced in the HG11 formulation than to the HG14 (37\% burst release in HG11 compared to $15 \%$ for HG14). This seemingly contradicts our previous data reporting both formulations having the same average pore size, in which case a similar degradation profile would be expected. However, HG14 median pore size is smaller than that of HG11, suggesting that even though there are a few large pores and cracks in the biomaterial, the majority of the pores are indeed smaller than in the HG1 1 formulation (7.6 $\mu \mathrm{m}$ for HG14 compared to $16.9 \mu \mathrm{m}$ for HG11) This supports the slower degradation observed initially, as well as the rapid disintegration of the hydrogel after a few days, when it breaks into macroscopic pieces owing to the effect of the larger pores.

We next assessed the viability of HDFs in contact with hydrogel degradation by-products released at different time intervals (0-24 hours, 24-72 hours and 72-168 hours). The starting reagents used to form the hydrogels (pBAE and 4-arm PEG) were also dissolved separately in culture medium at the concentration used for the hydrogel preparation. Cell viability experiments showed no significant cytotoxicity from the degradation by-products of HG11 collected after 24, 72 or 168 hours (Figure 4L, triangles). By contrast, HG14 degradation by-products released in the first 24 hours caused approximately 75\% HDF toxicity, while no toxicity was observed when cells were exposed to degradation products released after 24 hours (Figure 4L, squares). It is important to highlight that cells exposed to degradation by-products at 72 hours included only byproducts from the 24-72-hour window, but not the initial 0-24 hour window by-products, and the same applies for the time point at 168 hours, which contained only the 72-168-hour by-products. Hence, the behaviour observed suggests that the initial burst release from HG14 leads to a high 
enough concentration of by-products in the media to elicit fibroblast toxicity. Even though we reported lower burst release for HG14 compared to HG11 in relative numbers (\% pBAE released of total pBAE), HG14 contains 4-fold higher pBAE content than HG11, leading to overall higher concentration of by-products in the media after the initial burst release, causing higher toxicity. Indeed, the toxicity of the individual hydrogel components revealed that, while PEG-SH causes no significant cell death compared to the negative control, pBAEs are toxic at high concentrations (Figure 4L, white dots). Altogether, the data supports the hypothesis that the burst release of pBAE polymers from HG14 is toxic to dermal fibroblasts, while HG11 shows no significant toxicity, hence making this formulation more suited for use as a dermal wound dressing for the release of therapeutics. 

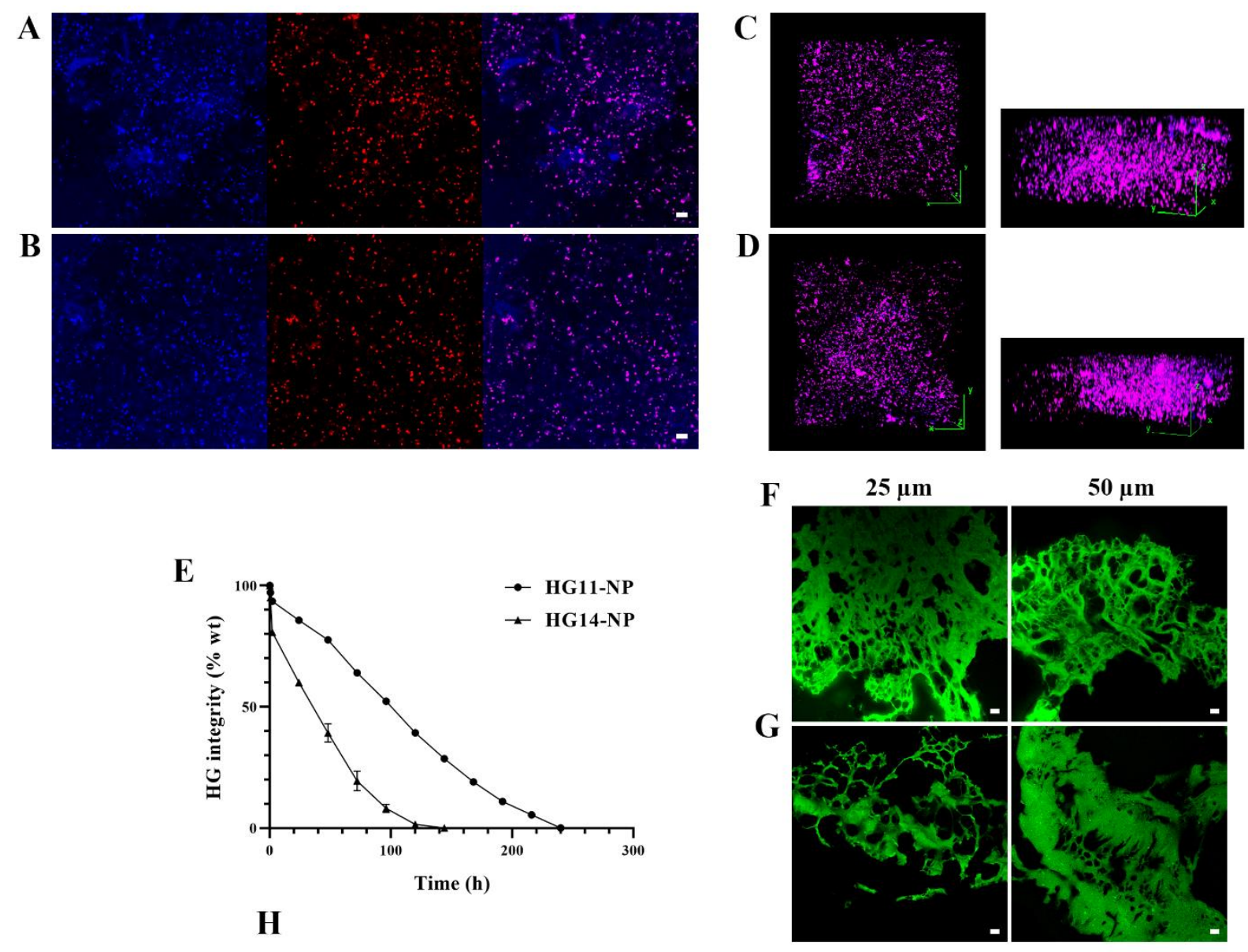

H
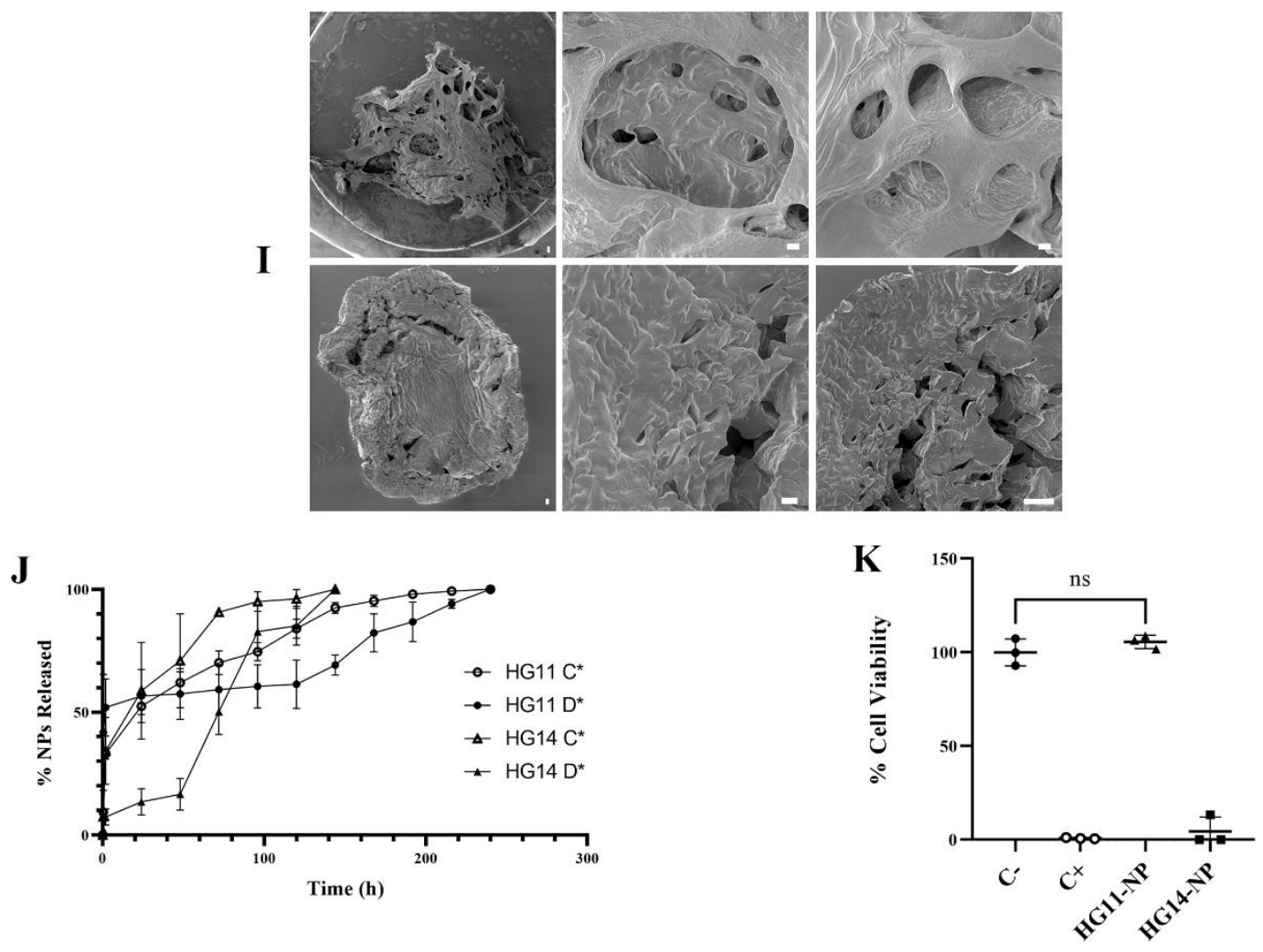
Figure 5. A) Confocal microscopy images of C6RH polyplexes loaded into hydrogel HG11 (cy5tagged pBAE shell: blue channel; cy3-tagged DNA core: red channel. Purple results from the pBAE and DNA signal overlap). B) Confocal microscopy images of C6RH polyplexes loaded into hydrogel HG14 (same tags and channels than that used in A)). A) and B) Scale bar: $100 \mu \mathrm{m} . \mathrm{C}$ ) Three-dimensional construction of a 79- $\mu \mathrm{m}$ thickness section of HG11 doped with C6RH-cy5 encapsulating pGFP-cy3. D) Three-dimensional construction of a $57-\mu \mathrm{m}$ thickness section of HG14 doped with C6RH-cy5 encapsulating pGFP-cy3. E) Degradation of hydrogels HG11 and HG14 loaded with polyplexes was tracked using fluorescently labelled pBAE, which was converted to weight $\%$ of pBAE in the hydrogel as a measure of hydrogel integrity; $\mathrm{n}=2$. F) and G) Confocal microscopy images of $25 \mu \mathrm{m}$ and $50 \mu \mathrm{m}$ slices of FITC-tagged C6RH-loaded HG11 and HG14 respectively. F) and G) scale bar: $50 \mu \mathrm{m}$. H and I) SEM images of lyophilized bulk C6RH-loaded hydrogels HG11 and HG14, respectively; scale bar: $50 \mu \mathrm{m}$. J) Release of fluorescently labelled C6RH nanoparticles from the hydrogels HG11 and HG14. C* corresponds to nanoparticles with cy3-tagged $\mathrm{pBAE}$ (shell) and $\mathrm{D}^{*}$ to nanoparticles with cy3-tagged DNA (core); $\mathrm{n}=2$. K) Cytotoxicity (in \% cell viability) of C6RH-loaded HG11 and HG14 after $24 \mathrm{~h}$ transfection.

Characterization of C6RH-loaded composite hydrogels. C6RH nanoparticles were incorporated into both hydrogel formulations (HG11 and HG14) to attain sustained local release and prolonged HDFs transfection. First, we studied the stability and distribution of nanoparticles within the hydrogel via confocal microscopy, where $2 \%$ of C6RH polymer and $10 \%$ of pGFP were tagged with cy5 and cy3, respectively. Fluorescence co-localization was used as a surrogate measurement of particle stability, revealing that tagged C6RH (blue) and pGFP (red) co-localized with a Pearson's R value of 0.97 and 0.90 (Figure 5A-B for HG11 and HG14, respectively). Manders' overlap coefficient, more accurate for images with different intensities ${ }^{56,57}$, also revealed high co-localization of the fluorescent signals (HG11-NP: 0.91 and 1.00; HG14-NP: 0.98 and 0.84 for channels 1 and 2, respectively). High co-localization of C6RH polymers and plasmid indicates that the nanoparticles formed are stable within the hydrogel structure, meaning they do not degrade or become undone through electrostatic interactions with PBAEs in the hydrogel). Threedimensional rendering of the hydrogel images showed that polyplexes were homogeneously distributed throughout the volume of the hydrogel in both HG11 (Figure 5C) and HG14 (Figure 5D) formulations.

Kinetics of pBAE polyplexes release from the hydrogels. Fluorescein-labelled HG11 and HG14 loaded with pBAE polyplexes (HG11-NP and HG14-NP) were prepared and degradation experiments were performed as described above. The release of polyplexes was also fluorescently tracked by labelling either the C6RH polymer shell or the encapsulated pGFP with cy3 at concentrations of $1 \%$ and $10 \%$, respectively. The addition of nanoparticles had a different impact on the overall degradation profiles of both formulations. While complete degradation of HG11NP occurred over a longer time-scale than that of HG11 (240 hours for HG11-NP versus 200 hours for HG11), HG14-NP degraded completely in almost a third of the time in comparison to HG14 
(144 hours compared to 360 hours, Figure 5E). Interestingly, the addition of nanoparticles to the HG11 formulation leads to the elimination of the initial burst release and a close to zero-order degradation kinetics. To eliminate a dilution factor as the cause of the unexpected behaviour of HG14, we studied the degradation of new formulations with equal final volumes (referred as HG14-500v and HG14-500v-NP). Despite having different final volumes (and hence reactants concentrations), HG14-500v presented similar degradation profile than HG14 (Supplementary figure S8), suggesting that the unexpected degradation kinetics were not caused by a dilution factor when adding the nanoparticles.

We then tested the effect of adding polyplexes to these new formulations (maintaining the final volume fixed). The data revealed the same unexpected acceleration of release kinetics previously observed in HG14-NP samples. Hence, the unexpected behaviour of HG14-NP is not due to a dilution factor caused by the addition of nanoparticles solution to the hydrogel mix but rather to the nanoparticles themselves interacting unexpectedly with the hydrogel network. We also explored whether decreasing the concentration of nanoparticles by half could restore the degradation profile of HG14-500v. Once again, the degradation kinetics of this sample were accelerated compared to the hydrogel formulation without nanoparticles, and followed similar profile than that containing double the amount of polyplexes. Given that this phenomenon is not observed in HG11-NP (containing 4-fold less molar ratio of pBAE), it is reasonable to hypothesize that the interactions of the excess of $\mathrm{C}_{32} \mathrm{~T}_{2} \mathrm{CR} 3 \mathrm{pBAE}$ in the hydrogel with $\mathrm{C} 6 \mathrm{RH}$ pBAE in the nanoparticles might be the cause of the hydrogel network destabilization and enhanced degradation kinetics. Indeed, both HG14-NP and HG14-500v-NP experienced visible macroscopic holes and started to break into pieces after 48 hours, leading to an acceleration of the degradation. This also correlates with the increased fragility of HG14 formulation from our rheological studies. Fluorescence microscopy analysis of cryosectioned HG14-NP samples also revealed pores are not distributed in a uniform manner but forming cracks through the hydrogel (Figure 5G), as opposed to the more uniform pore size distribution of HG11-NP samples (Figure 5F and Supplementary figure S9). SEM image analysis of whole, lyophilized hydrogels corroborated these findings and confirmed that the observed cracks and large pores were not artefacts of the mechanical cryosectioning process (Figure 5H, I).

The unexpected behaviour of HG14-NP formulation was also observed in the polyplex release experiments. Both C6RH forming the nanoparticle shell and encapsulated DNA were released following similar kinetics from HG11-NP samples (Figure 5J, circles), suggesting that the initial nanoparticle stability observed in confocal microscopy is maintained over time and after delivery. Contrarily, most of the C6RH pBAE forming the nanoparticles loaded in HG41-NP was quickly and steadily released within 72 hours, while the DNA remained within the hydrogel for the first 48 hours, and then was quickly released in the following 48 hours (Figure 5J, triangles). This points towards polyplexes' disruption after hydrogel formation, followed by quick release of $\mathrm{C} 6 \mathrm{RH}$ owing to electrostatic repulsion with $\mathrm{C}_{3} 2 \mathrm{~T}_{2} \mathrm{CR} 3$ polymers forming the hydrogel network. Negatively charged DNA can then interact with positively charged $\mathrm{C}_{32} \mathrm{~T}_{2} \mathrm{CR} 3$ hydrogel network 
and be released with the bulk material as it fractures after 48 hours. This indicates that, as inferred above from the degradation data, high $\mathrm{C}_{32} \mathrm{~T}_{2} \mathrm{CR} 3$ concentrations can interact adversely with C6RH polymers, leading to the destabilization of both the hydrogel network and the nanoparticles structure. Further research, beyond the scope of this manuscript, is needed to confirm our hypothesis that the underlying mechanism is based on the repulsive forces between positively charged polymers.

Before quantifying the transfection efficiency of mRNA-loaded polyplexes released from the hydrogel formulations in HDFs, a cytotoxicity assay of the composite material in direct contact with cells was performed. The viability of fibroblasts treated with the formulation HG11 did not show any significant difference compared to untreated fibroblast (Figure 5K), consistent with previously conducted cytotoxicity studies using the hydrogel's degradation products (without polyplexes). In contrast, cells treated with the formulation HG14-NP presented a cell viability comparable to that of the positive control, revealing again that the increase of $\mathrm{pBAE}$ in the formulation causes high cytotoxicity.

A
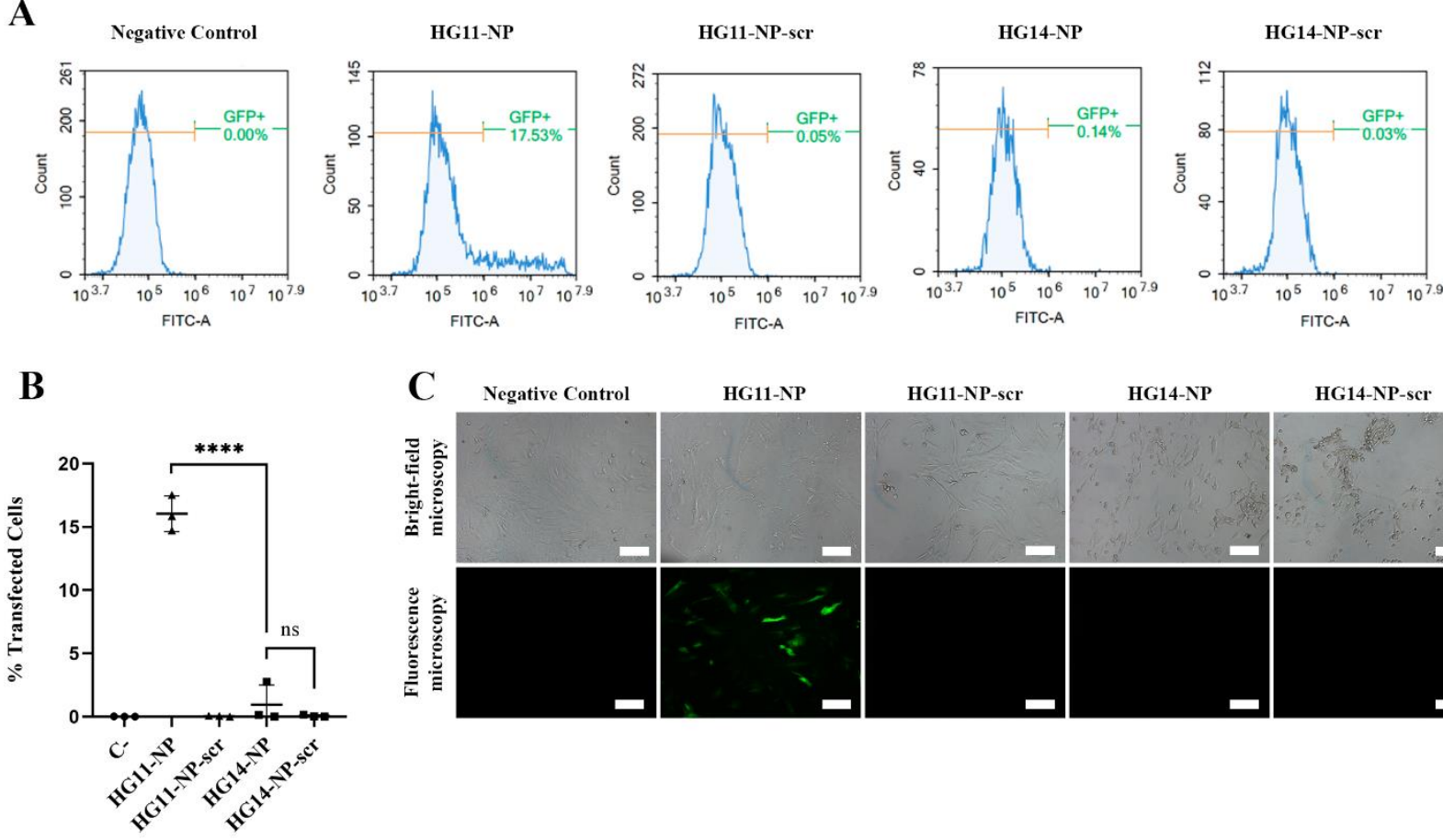

C Negative Contro

HG11-NP
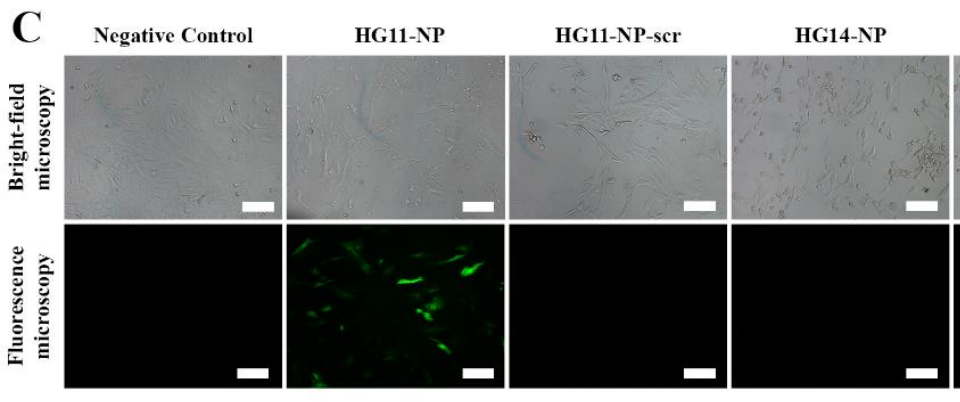

HG14-NP-scr

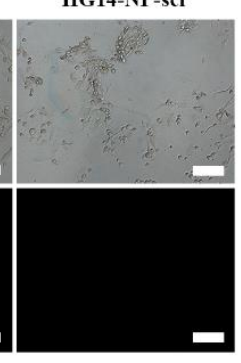

Figure 6. A) FACS graphs showing the percentage of single HDF cells counted expressing GFP after transfection using the C6RH-loaded polyplexes encapsulating mRNA-GFP or a scrambled RNA. B) Percentage of transfected cells after $24 \mathrm{~h}$ in contact with the hydrogel formulations studied. C) Bright-field and fluorescence microscopy images of HDFs after $24 \mathrm{~h}$ contact with the hydrogel formulations used in the transfection experiment. Scale bar: $100 \mu \mathrm{m} . \mathrm{n}=3$. ${ }^{*} \mathrm{p}<0.05$; $* * \mathrm{p}<0.01 ; * * * \mathrm{p}<0.001 ; * * * * \mathrm{p}<0.0001$. 
Transfection efficiency of hydrogels doped with mRNA-GFP-loaded C6RH polyplexes in HDFs. According to the data obtained in the release experiments, HG11-NP and HG14-NP hydrogels were doped with the necessary amount of polyplexes to release the same quantity of mRNA-GFP per cell when compared to the transfection experiments after $24 \mathrm{~h}$ transfection (Figure 5J). A scrambled mRNA sequence encapsulated in C6RH and loaded into both hydrogel formulations served as negative controls, as well as non-transfected cells. HG11-NP formulation showed a significant difference in mRNA-GFP transfection compared to the same formulation encapsulating scrambled RNA as genetic material, with roughly $20 \%$ of HDF expressing GFP (Figure 6A-B). The transfection efficiency is lower than that observed when we incubate cells with free nanoparticles (without hydrogel release system). This is to be expected given that cells are exposed to a lower nanoparticle concentration throughout the experiment owing to the sustained release. At the time of fluorescence measurement, not all released nanoparticles have been uptaken by cells and not all uptaken nanoparticles have been translated into green fluorescence protein. Moreover, previous studies report that GFP protein expression progressively increases in the 24 hours following mRNA transfection, and that there is an inherently large cellto-cell variability in the expression levels of GFP of transfected cells ${ }^{58}$. Hence, only those cells transfected in the first few hours are expected to emit a significant amount of fluorescence at the time of measurement.

Interestingly, the green fluorescence expression of HDFs transfected with GFP-mRNA-loaded HG14-NP was not significantly different from that of the same hydrogel loaded with scrambled mRNA, indicating that no transfection takes place with these formulations (Figure 6A-B). This aligns with the data gathered in the release experiments, where the genetic material and the $\mathrm{C} 6 \mathrm{RH}$ polymer forming the polyplexes were released with different kinetics, and supports our hypothesis that polyplexes loaded into HG14 hydrogels degrade after a few hours, rendering them nonfunctional as transfection reagents. Hence, the formulation we herein name HG11-NP shows the capability to transfect primary dermal cells with a relatively high efficiency without compromising cell viability, and hence has high potential as an injectable wound dressing for gene therapy of chronic and delayed-healing wounds.

\section{CONCLUSIONS}

Gene therapy is rapidly gaining traction in our society, especially with the recent approval of mRNA vaccines. There is a pressing need for new and improved transfection reagents along with suitable delivery vehicles that can be easily translated into clinical therapeutic approaches to deliver genetic material to all types of cells. While a significant effort has been placed into developing nanotechnology for the transfection of tumour cells, the viable and efficacious options for primary human cells are limited. Cutaneous chronic wounds display altered gene expression and mRNA dysregulation and could benefit from gene therapy. However, their irregular topology often makes it difficult to deliver any therapeutic in a sustained and controlled manner. In this work, we have developed a prototype of wound dressing based on a viscoelastic hydrogel made of 
poly( $\beta$-amino ester)s and PEG polymers. We have designed this wound dressing to be biodegradable, biocompatible and, most importantly, injectable. The latter property allows the material to be applied in liquid form and gel in situ to adapt to irregular, deep wounds such as chronic ulcers. We have also shown that this novel hydrogel is suitable as a depot for controlled delivery of pBAE nanoparticles loaded with genetic material over a variable time frame (8 to 15 days depending on the formulation). As a proof of concept, the nanoparticles developed herein were shown to efficiently transfect human dermal fibroblasts with both mRNA and DNA encoding for green fluorescent protein. Particle stability and transfection efficiency were maintained after release from the hydrogel, demonstrating that this prototype wound dressing is an ideal candidate for gene therapy applied to cutaneous chronic wound healing. These results lay the groundwork for future studies exploring the therapeutic potential of the platform using preclinical models of defective wound healing. Further studies will aim at validating the transfection efficiency of these pBAE nanoparticles in other human primary cell types (i.e. endothelial cells, osteocytes, cardiomyocytes) to generalize the use of this platform for improving impaired healing or simply accelerating normal healing after surgery or trauma, in turn reducing the length of hospitalization and accelerating the return to work.

\section{ASSOCIATED CONTENT}

The following files are available free of charge.

Supporting Information. Figure S1, characterization of polymer $\mathrm{C}_{2} 2 \mathrm{~T}_{2}$ by ${ }^{1} \mathrm{H}-\mathrm{NMR}$; Figure S2, characterization of polymer $\mathrm{C}_{32} \mathrm{~T}_{2} \mathrm{CR} 3$ by ${ }^{1} \mathrm{H}-\mathrm{NMR}$; Table S1, nanoparticle hydrodynamic diameter and PdI of the formulations studied; Figure S3, transfection efficiency using GFP-coding polynucleotides; Figure S4, transfection efficiency using luciferase-coding polynucleotides; Figure S5, evidence on hydrogel formation via disulphide coupling by ${ }^{1} \mathrm{H}-\mathrm{NMR}$; Figure S6, swelling ratios of the hydrogel formulations studied. Figure S7, pore size distribution in HG11 and HG14; Figure S8, degradation of hydrogels HG14 and HG14-500v; Figure S9, pore size distribution in formulations HG11, HG14, HG11-NP and HG14-NP (PDF)

\section{AUTHOR INFORMATION}

\section{Corresponding Authors}

Salvador Borrós - Grup d'Enginyera de Materials (GEMAT), Institut Químic de Sarrià, Universitat Ramon Llull, via Augusta 390, 08017 Barcelona, Spain.

Nuria Oliva - Department of Bioengineering, Imperial College London, London SW7 2AZ, United Kingdom

\section{Authors}

José Antonio Durán-Mota - Grup d'Enginyera de Materials (GEMAT), Institut Químic de Sarrià, Universitat Ramon Llull, via Augusta 390, 08017 Barcelona, Spain. 
Júlia Quintanas Yani - Grup d'Enginyera de Materials (GEMAT), Institut Químic de Sarrià, Universitat Ramon Llull, via Augusta 390, 08017 Barcelona, Spain.

Benjamin D. Almquist - Department of Bioengineering, Imperial College London, London SW7 2AZ, United Kingdom

\section{AUTHOR CONTRIBUTIONS}

SB and NO conceived the project and designed the experiments. JADM and JQY designed and performed the experiments, collected and analyzed the data. BDA provided mentorship and advice. BDA, SB and NO secured the funding for the research. JADM, SB and NO co-wrote the manuscript. All authors analyzed and discussed the results and reviewed the manuscript. All authors approve the final version of the manuscript.

\section{ACKNOWLEDGMENTS}

N.O. acknowledges funding from the European Union's Horizon 2020 research and innovation programme under the Marie Sklodowska-Curie grant agreement No 712949 (TECNIOspring PLUS) and from the Agency for Business Competitiveness of the Government of Catalonia, along with funding from an Imperial College Research Fellowship. S.B. acknowledges funding from the Ministerio de Ciencia, Innovación y Universidades, project number RTI2018-094734B-C22. B.D.A acknowledges funding from the Engineering and Physical Sciences Research Council, project number EP/R041628/1. We thank Dr. Meysam Keshavarz from the Hamlyn Centre at Imperial College London for assistance with SEM imaging and Miguel Hermida Ayala from the Department of Bioengineering at Imperial College London and Cristina Fornaguera Puigvert from the Department of Bioengineering at Institut Químic de Sarrià for assistance with confocal microscopy imaging. We also want to thank Núria Agulló Chaler for her knowledge and assistance with the rheometry experiments and Marta Guerra Rebollo for assistance with the luciferase mRNA transfection experiments.

\section{ABBREVIATIONS}

4arm-PEG-SH, thiolated 4arm-polyethylene glycol; FACS, Fluorescence-activated Cell Sorting; HDFs, human dermal fibroblasts; OM-pBAEs, oligopeptide-modified poly( $\beta$-amino esters)s; pBAEs, poly( $\beta$-amino esters)s; SPDP, 3-(2-pyridyldithio)propanoic acid.

\section{REFERENCES}

(1) Werner, S.; Grose, R. Regulation of Wound Healing by Growth Factors and Cytokines. Physiol. Rev. 2003, 83 (3), 835-870. https://doi.org/10.1152/physrev.2003.83.3.835. 
(2) Singer, A. J.; Clark, R. A. F. Cutaneous Wound Healing. N. Engl. J. Med. 1999, 341 (10), 738-746. https://doi.org/10.1056/NEJM199909023411006.

(3) Guo, S.; DiPietro, L. A. Factors Affecting Wound Healing. J. Dent. Res. 2010, 89 (3), 219229. https://doi.org/10.1177/0022034509359125.

(4) Sen, C. K. Human Wounds and Its Burden: An Updated Compendium of Estimates. Adv. Wound Care 2019, 8 (2), 39-48. https://doi.org/10.1089/wound.2019.0946.

(5) Eming, S. A.; Martin, P.; Tomic-Canic, M. Wound Repair and Regeneration: Mechanisms, Signaling, and Translation. Sci. Transl. Med. 2014, $6 \quad$ (265), $265 \mathrm{sr} 6$. https://doi.org/10.1126/scitranslmed.3009337.

(6) Broughton, G.; Janis, J. E.; Attinger, C. E. Wound Healing: An Overview. Plast. Reconstr. Surg. $\quad 2006, \quad 117 \quad$ (SUPPLEMENT), https://doi.org/10.1097/01.prs.0000222562.60260.f9.

(7) Morton, L. M.; Phillips, T. J. Wound Healing and Treating Wounds. J. Am. Acad. Dermatol. 2016, 74 (4), 589-605. https://doi.org/10.1016/j.jaad.2015.08.068.

(8) Han, G.; Ceilley, R. Chronic Wound Healing: A Review of Current Management and Treatments. Adv. Ther. 2017, 34 (3), 599-610. https://doi.org/10.1007/s12325-017-0478-y.

(9) van de Vyver, M.; Niesler, C.; Myburgh, K. H.; Ferris, W. F. Delayed Wound Healing and Dysregulation of IL6/STAT3 Signalling in MSCs Derived from Pre-Diabetic Obese Mice. Mol. Cell. Endocrinol. 2016, 426, 1-10. https://doi.org/10.1016/j.mce.2016.02.003.

(10) Takematsu, E.; Spencer, A.; Auster, J.; Chen, P.-C.; Graham, A.; Martin, P.; Baker, A. B. Genome Wide Analysis of Gene Expression Changes in Skin from Patients with Type 2 Diabetes. PLoS One 2020, 15 (2), e0225267. https://doi.org/10.1371/journal.pone.0225267.

(11) Layliev, J.; Wilson, S.; Warren, S. M.; Saadeh, P. B. Improving Wound Healing with Topical Gene Therapy. Adv. Wound Care 2012, 1 (5), 218-223. https://doi.org/10.1089/wound.2011.0322.

(12) Oryan, A.; Alemzadeh, E.; Zarei, M. Basic Concepts, Current Evidence, and Future Potential for Gene Therapy in Managing Cutaneous Wounds. Biotechnol. Lett. 2019, 41 (89), 889-898. https://doi.org/10.1007/s10529-019-02701-6.

(13) Khalil, A. S.; Yu, X.; Umhoefer, J. M.; Chamberlain, C. S.; Wildenauer, L. A.; Diarra, G. M.; Hacker, T. A.; Murphy, W. L. Single-Dose MRNA Therapy via Biomaterial-Mediated Sequestration of Overexpressed Proteins. Sci. Adv. 2020, 6 (27), eaba2422. https://doi.org/10.1126/sciadv.aba2422.

(14) Sun, N.; Ning, B.; Hansson, K. M.; Bruce, A. C.; Seaman, S. A.; Zhang, C.; Rikard, M.; 
DeRosa, C. A.; Fraser, C. L.; Wågberg, M.; Fritsche-Danielson, R.; Wikström, J.; Chien, K. R.; Lundahl, A.; Hölttä, M.; Carlsson, L. G.; Peirce, S. M.; Hu, S. Modified VEGF-A MRNA Induces Sustained Multifaceted Microvascular Response and Accelerates Diabetic Wound Healing. Sci. Rep. 2018, 8 (1), 17509-17519. https://doi.org/10.1038/s41598-01835570-6.

(15) Khalil, I. A.; Sato, Y.; Harashima, H. Recent Advances in the Targeting of Systemically Administered Non-Viral Gene Delivery Systems. Expert Opin. Drug Deliv. 2019, 16 (10), 1037-1050. https://doi.org/10.1080/17425247.2019.1656196.

(16) Nayerossadat, N.; Ali, P.; Maedeh, T. Viral and Nonviral Delivery Systems for Gene Delivery. Adv. Biomed. Res. 2012, 1 (2), 27-37. https://doi.org/10.4103/2277-9175.98152.

(17) Hirsch, T.; Spielmann, M.; Yao, F.; Eriksson, E. Gene Therapy in Cutaneous Wound Healing. Front. Biosci. 2007, 12 (7), 2507-2518. https://doi.org/10.2741/2251.

(18) Wu, P.; Chen, H.; Jin, R.; Weng, T.; Ho, J. K.; You, C.; Zhang, L.; Wang, X.; Han, C. NonViral Gene Delivery Systems for Tissue Repair and Regeneration. J. Transl. Med. 2018, 16 (1), 29-48. https://doi.org/10.1186/s12967-018-1402-1.

(19) Aggarwal, R.; Targhotra, M.; Kumar, B.; Sahoo, P. ; Chauhan, M. K. Polyplex: A Promising Gene Delivery System. Int. J. Pharm. Sci. Nanotechnol. 2019, 12 (6), 46814686. https://doi.org/10.37285//ijpsn.2019.12.6.1.

(20) Kozielski, K. L.; Tzeng, S. Y.; Hurtado De Mendoza, B. A.; Green, J. J. Bioreducible Cationic Polymer-Based Nanoparticles for Efficient and Environmentally Triggered Cytoplasmic SiRNA Delivery to Primary Human Brain Cancer Cells. ACS Nano 2014, 8 (4), 3232-3241. https://doi.org/10.1021/nn500704t.

(21) Segovia, N.; Dosta, P.; Cascante, A.; Ramos, V.; Borrós, S. Oligopeptide-Terminated Poly(ß-Amino Ester)s for Highly Efficient Gene Delivery and Intracellular Localization. Acta Biomater. 2014, 10 (5), 2147-2158. https://doi.org/10.1016/j.actbio.2013.12.054.

(22) Tzeng, S. Y.; Hung, B. P.; Grayson, W. L.; Green, J. J. Cystamine-Terminated Poly(BetaAmino Ester)s for SiRNA Delivery to Human Mesenchymal Stem Cells and Enhancement of Osteogenic Differentiation. Biomaterials 2012, 33 (32), 8142-8151. https://doi.org/10.1016/j.biomaterials.2012.07.036.

(23) Tzeng, S. Y.; Green, J. J. Subtle Changes to Polymer Structure and Degradation Mechanism Enable Highly Effective Nanoparticles for SiRNA and DNA Delivery to Human Brain Cancer. Adv. Healthc. Mater. 2013, 2 (3), 468-480. https://doi.org/10.1002/adhm.201200257.

(24) Zugates, G. T.; Peng, W.; Zumbuehl, A.; Jhunjhunwala, S.; Huang, Y. H.; Langer, R.; 
Sawicki, J. A.; Anderson, D. G. Rapid Optimization of Gene Delivery by Parallel EndModification of Poly( $\beta$-Amino Ester)S. Mol. Ther. 2007, 15 (7), 1306-1312. https://doi.org/10.1038/sj.mt.6300132.

(25) Ahmad, A.; Khan, J. M.; Haque, S. Strategies in the Design of Endosomolytic Agents for Facilitating Endosomal Escape in Nanoparticles. Biochimie 2019, 160, 61-75. https://doi.org/10.1016/j.biochi.2019.02.012.

(26) Keeney, M.; Ong, S. G.; Padilla, A.; Yao, Z.; Goodman, S.; Wu, J. C.; Yang, F. Development of Poly( $\beta$-Amino Ester)-Based Biodegradable Nanoparticles for Nonviral Delivery of Minicircle DNA. ACS Nano 2013, 7 (8), 7241-7250. https://doi.org/10.1021/nn402657d.

(27) Dosta, P.; Ramos, V.; Borrós, S. Stable and Efficient Generation of Poly( $\beta$-Amino Ester)s for RNAi Delivery. Mol. Syst. Des. Eng. 2018, 3 (4), 677-689. https://doi.org/10.1039/c8me00006a.

(28) Meenach, S. A.; Otu, C. G.; Anderson, K. W.; Hilt, J. Z. Controlled Synergistic Delivery of Paclitaxel and Heat from Poly( $\beta$-Amino Ester)/Iron Oxide-Based Hydrogel Nanocomposites. Int. J. Pharm. 2012, $427 \quad$ (2), 177-184. https://doi.org/10.1016/j.ijpharm.2012.01.052.

(29) Perni, S.; Prokopovich, P. Poly-Beta-Amino-Esters Nano-Vehicles Based Drug Delivery System for Cartilage. Nanomedicine Nanotechnology, Biol. Med. 2017, 13 (2), 539-548. https://doi.org/10.1016/j.nano.2016.10.001.

(30) Tzeng, S. Y.; Guerrero-Cázares, H.; Martinez, E. E.; Sunshine, J. C.; Quiñones-Hinojosa Alfredo, A.; Green, J. J. Non-Viral Gene Delivery Nanoparticles Based on Poly( $\beta$-Amino Esters) for Treatment of Glioblastoma. Biomaterials 2011, 32 (23), 5402-5410. https://doi.org/10.1016/j.biomaterials.2011.04.016.

(31) Segovia, N.; Pont, M.; Oliva, N.; Ramos, V.; Borrós, S.; Artzi, N. Hydrogel Doped with Nanoparticles for Local Sustained Release of SiRNA in Breast Cancer. Adv. Healthc. Mater. 2014, 4 (2), 271-280. https://doi.org/10.1002/adhm.201400235.

(32) Oliva, N.; Conde, J.; Wang, K.; Artzi, N. Designing Hydrogels for On-Demand Therapy. Acc. Chem. Res. 2017, 50 (4), 669-679. https://doi.org/10.1021/acs.accounts.6b00536.

(33) Boateng, J. S.; Matthews, K. H.; Stevens, H. N. E.; Eccleston, G. M. Wound Healing Dressings and Drug Delivery Systems: A Review. J. Pharm. Sci. 2008, 97 (8), 2892-2923. https://doi.org/10.1002/jps.21210.

(34) Oliva, N.; Almquist, B. D. Spatiotemporal Delivery of Bioactive Molecules for Wound Healing Using Stimuli-Responsive Biomaterials. Adv. Drug Deliv. Rev. 2020, 161-162, 22- 
41. https://doi.org/10.1016/j.addr.2020.07.021.

(35) Jones, A.; Vaughan, D. Hydrogel Dressings in the Management of a Variety of Wound Types: A Review. J. Orthop. Nurs. 2005, 9 (SUPPL. 1), S1-S11. https://doi.org/10.1016/S1361-3111(05)80001-9.

(36) Koehler, J.; Brandl, F. P.; Goepferich, A. M. Hydrogel Wound Dressings for Bioactive Treatment of Acute and Chronic Wounds. Eur. Polym. J. 2018, 100, 1-11. https://doi.org/10.1016/j.eurpolymj.2017.12.046.

(37) Kamoun, E. A.; Kenawy, E. R. S.; Chen, X. A Review on Polymeric Hydrogel Membranes for Wound Dressing Applications: PVA-Based Hydrogel Dressings. J. Adv. Res. 2017, 8 (3), 217-233. https://doi.org/10.1016/j.jare.2017.01.005.

(38) Sharman, D. Moist Wound Healing: A Review of Evidence, Application and Outcome. Diabet. Foot J. 2003, 6 (3), 112-116 3p.

(39) Sen, C. K. Wound Healing Essentials: Let There Be Oxygen. Wound Repair Regen. 2009, 17 (1), 1-18. https://doi.org/10.1111/j.1524-475X.2008.00436.x.

(40) Cascone, S.; Lamberti, G. Hydrogel-Based Commercial Products for Biomedical Applications: A Review. Int. J. Pharm. 2020, 573, 118803-118821. https://doi.org/10.1016/j.jpharm.2019.118803.

(41) Schreml, S.; Szeimies, R.-M.; Prantl, L.; Landthaler, M.; Babilas, P. Wound Healing in the 21st Century. J. Am. Acad. Dermatol. 2010, 63 (5), 866-881. https://doi.org/10.1016/j.jaad.2009.10.048.

(42) Berberich, B.; Thriene, K.; Gretzmeier, C.; Kühl, T.; Bayer, H.; Athanasiou, I.; RafeiShamsabadi, D. A.; Bruckner-Tuderman, L.; Nyström, A.; Kiritsi, D.; Dengjel, J. Proteomic Profiling of Fibroblasts Isolated from Chronic Wounds Identifies Disease-Relevant Signaling Pathways. J. Invest. Dermatol. 2020, 140 (11), 2280-2290.e4. https://doi.org/10.1016/j.jid.2020.02.040.

(43) Darby, I. A.; Laverdet, B.; Bonté, F.; Desmoulière, A. Clinical, Cosmetic and Investigational Dermatology Dovepress Fibroblasts and Myofibroblasts in Wound Healing. Clin. Cosmet. Investig. Dermatol. 2014, 4 (7), 301-311.

(44) Kim, B. C.; Kim, H. T.; Park, S. H.; Cha, J. S.; Yufit, T.; Kim, S. J.; Falanga, V. Fibroblasts from Chronic Wounds Show Altered TGF- $\beta$-Signaling and Decreased TGF- $\beta$ Type II Receptor Expression. J. Cell. Physiol. 2003, 195 (3), 331-336. https://doi.org/10.1002/jcp.10301.

(45) Madhyastha, R.; Madhyastha, H.; Nakajima, Y.; Omura, S.; Maruyama, M. MicroRNA Signature in Diabetic Wound Healing: Promotive Role of MiR-21 in Fibroblast Migration. 
Int. Wound J. 2012, 9 (4), 355-361. https://doi.org/10.1111/j.1742-481X.2011.00890.x.

(46) Fornaguera, C.; Guerra-Rebollo, M.; Ángel Lázaro, M.; Castells-Sala, C.; Meca-Cortés, O.; Ramos-Pérez, V.; Cascante, A.; Rubio, N.; Blanco, J.; Borrós, S. MRNA Delivery System for Targeting Antigen-Presenting Cells In Vivo. Adv. Healthc. Mater. 2018, 7 (17), 1-11. https://doi.org/10.1002/adhm.201800335.

(47) Dosta, P.; Segovia, N.; Cascante, A.; Ramos, V.; Borrós, S. Surface Charge Tunability as a Powerful Strategy to Control Electrostatic Interaction for High Efficiency Silencing, Using Tailored Oligopeptide-Modified Poly(Beta-Amino Ester)s (PBAEs). Acta Biomater. 2015, 20, 82-93. https://doi.org/10.1016/j.actbio.2015.03.029.

(48) Khalil, I. A.; Futaki, S.; Niwa, M.; Baba, Y.; Kaji, N.; Kamiya, H.; Harashima, H. Mechanism of Improved Gene Transfer by the N-Terminal Stearylation of Octaarginine: Enhanced Cellular Association by Hydrophobic Core Formation. Gene Ther. 2004, 11 (7), 636-644. https://doi.org/10.1038/sj.gt.3302128.

(49) Takigawa, D. Y.; Tirrell, D. A. Disruption of Phospholipid Packing by Branched Poly(Ethylenimine) Derivatives. Macromolecules 1985, 18 (3), 338-342. https://doi.org/10.1021/ma00145a006.

(50) Rejman, J.; Oberle, V.; Zuhorn, I. S.; Hoekstra, D. Size-Dependent Internalization of Particles via the Pathways of Clathrin-and Caveolae-Mediated Endocytosis. Biochem. J. 2004, 377 (1), 159-169. https://doi.org/10.1042/BJ20031253.

(51) Prabha, S.; Zhou, W.-Z.; Panyam, J.; Labhasetwar, V. Size-Dependency of NanoparticleMediated Gene Transfection: Studies with Fractionated Nanoparticles. Int. J. Pharm. 2002, 244 (1-2), 105-115. https://doi.org/10.1016/S0378-5173(02)00315-0.

(52) Rajesh, S.; Jr., J. W. L. Nanoparticle-Based Targeted Drug Delivery. Exp. Mol. Pathol. 2000, 86 (3), 215-223. https://doi.org/10.1016/j.yexmp.2008.12.004.Nanoparticle-based.

(53) Kulkarni, S. A.; Feng, S. S. Effects of Particle Size and Surface Modification on Cellular Uptake and Biodistribution of Polymeric Nanoparticles for Drug Delivery. Pharm. Res. 2013, 30 (10), 2512-2522. https://doi.org/10.1007/s11095-012-0958-3.

(54) Faraji, A. H.; Wipf, P. Nanoparticles in Cellular Drug Delivery. Bioorg. Med. Chem. 2009, 17 (8), 2950-2962. https://doi.org/10.1016/j.bmc.2009.02.043.

(55) de Barros, A.; Tsourkas, A.; Saboury, B.; Cardoso, V.; Alavi, A. Emerging Role of Radiolabeled Nanoparticles as an Effective Diagnostic Technique. EJNMMI Res. 2012, 2 (1), 39-53. https://doi.org/10.1186/2191-219X-2-39.

(56) Adler, J.; Parmryd, I. Quantifying Colocalization by Correlation: The Pearson Correlation Coefficient Is Superior to the Mander's Overlap Coefficient. Cytom. Part A 2010, 77 (8), 
733-742. https://doi.org/10.1002/cyto.a.20896.

(57) Pike, J. A.; Styles, I. B.; Rappoport, J. Z.; Heath, J. K. Quantifying Receptor Trafficking and Colocalization with Confocal Microscopy. Methods 2017, 115, 42-54. https://doi.org/10.1016/j.ymeth.2017.01.005.

(58) Reiser, A.; Woschée, D.; Mehrotra, N.; Krzysztoń, R.; Strey, H. H.; Rädler, J. O. Correlation of MRNA Delivery Timing and Protein Expression in Lipid-Based Transfection. Integr. Biol. 2019, 11 (9), 362-371. https://doi.org/10.1093/intbio/zyz030. 